\title{
Article
}

\section{Dual Fire Retardant Action: The Combined Gas and Condensed Phase Effects of Azo-Modified NiZnAl Layered Double Hydroxide on Intumescent Polypropylene}

Wang, Peng-Ji, Hu, Xiao-Ping, Liao, Dui-Jun, Wen, Yi, Hull, T Richard, Miao, Fei and Zhang, Quan-Tong

Available at https://clok.uclan.ac.uk/17216/

Wang, Peng-Ji, Hu, Xiao-Ping, Liao, Dui-Jun, Wen, Yi, Hull, T Richard orcid iconORCID: 0000-0002-7970-4208, Miao, Fei and Zhang, Quan-Tong (2017) Dual Fire Retardant Action: The Combined Gas and Condensed Phase Effects of Azo-Modified NiZnAl Layered Double Hydroxide on Intumescent

Polypropylene. Industrial \& Engineering Chemistry Research, 56 (4). pp. 920 -

932. ISSN 0888-5885

It is advisable to refer to the publisher's version if you intend to cite from the work. http://dx.doi.org/10.1021/acs.iecr.6b03953

For more information about UCLan's research in this area go to http://www.uclan.ac.uk/researchgroups/ and search for <name of research Group>.

For information about Research generally at UCLan please go to http://www.uclan.ac.uk/research/

All outputs in CLoK are protected by Intellectual Property Rights law, including Copyright law. Copyright, IPR and Moral Rights for the works on this site are retained by the individual authors and/or other copyright owners. Terms and conditions for use of this material are defined in the policies page. 


\title{
Dual Fire Retardant Action: The Combined Gas and Condensed Phase Effects of Azo-modified NiZnAl Layered Double Hydroxide on Intumescent Polypropylene
}

Peng-Ji Wang ${ }^{\dagger}$, Xiao-Ping Hu ${ }^{*+*}$, Dui-Jun Liao ${ }^{\dagger}$ Yi Wen ${ }^{\dagger}$, T. Richard Hull ${ }^{*+}$, Fei Miao ${ }^{\dagger}$, QuanTong Zhang ${ }^{\dagger}$

'School of Materials Science and Engineering, Southwest University of Science and Technology, Mianyang 621010, P. R. China

${ }^{\ddagger}$ Centre for Fire and Hazards Science, University of Central Lancashire, Preston PR1 2HE, UK

\begin{abstract}
Ternary nickel-substituted layered double hydroxide (C-LDH) was synthesized. It was intercalated with azobenzene-4,4'-dicarboxylic acid, using an ion exchange method to obtain organically modified NiZnAl-LDH (O-LDH). Both LDHs were melt-blended into polypropylene (PP) with intumescent fire retardant (IFR). The structure, morphology, thermal stability and combustible properties of intercalated LDH and its hybrid composite have been comprehensively characterized. SEM and EDX mapping show O-LDH exhibits better dispersion than ZnNiAl$\mathrm{CO}_{3} \mathrm{LDH}(\mathrm{C}-\mathrm{LDH})$. Cone calorimetry shows the addition of IFR and LDH significantly reduced smoke and heat release rate. The composite with $1 \mathrm{wt} \% \mathrm{O}-\mathrm{LDH}$, which showed dual gas phase and condensed phase fire retardant action, exhibited the lowest flammability with an LOI value of $29.3 \%$ and achieved a UL-94 V-0 rating. In addition, incorporation of LDH improved the mechanical properties compared to PP/IFR composites. UV absorption showed that O-LDH could significantly improve the ultraviolet stability of PP composites.
\end{abstract}

\section{INTRODUCTION}

Polypropylene (PP) has been widely used in many fields including the packaging, electronics, automotive, building and chemical industry etc., due to its low cost, good mechanical properties, low toxicity, light weight and good processability ${ }^{1,2}$. However, in common with other hydrocarbon polymers, it is highly flammable and can produce large amounts of toxic smoke during combustion, both of which threaten the safety of human life and restrict its use in highrisk applications ${ }^{3}$. Various attempts have been made to reduce the flammability by incorporation of fire retardants. Traditional, halogen-based flame retardants have some significant drawbacks. Some are persistent, bio-accumulative and toxic (PBT) and have been banned or voluntarily 
withdrawn. As gas phase flame inhibitors, they increase the toxic smoke and gases (e.g CO, $\mathrm{HCl}$ and brominated dioxins) emitted ${ }^{4}$. Inorganic flame retardants such as $\mathrm{Mg}(\mathrm{OH})_{2}$ and $\mathrm{Al}(\mathrm{OH})_{3}$ require a high loading to achieve the required fire retardance ${ }^{5}$. However, adding a large amount will adversely affect the mechanical properties of the polymeric material ${ }^{6}$. In recent years, intumescent flame retardants (IFRs) have attracted more and more attention in flame-retarding polypropylene (PP) because of their halogen-free, environmental-friendly, low smoke, and low toxicity properties ${ }^{7-12}$. IFR usually consists of three ingredients: acid source, carbonization agent and blowing agent. IFR can form a foamed char layer during the early stages of burning, which acts as a physical barrier, slowing heat and mass transfer between the burning zone and polymer matrix ${ }^{14}$. However, the traditional IFR has some drawbacks, such as forming a mechanically weak, non-compact and thermally unstable char layer, a weaker polymer resulting from the high loading needed, and poor compatibility with polymer matrix. In general, an additional synergistic agent is one of the most effective methods to address these disadvantages.

The current synergistic agents used in IFR include zinc borates ${ }^{13}$, metal oxides, layerstructured montmorillonites ${ }^{14}$, sepiolite ${ }^{15}$, carbon nanotubes ${ }^{16}$, graphene, etc. In recent years, layered double hydroxides (LDHs), also known as hydrotalcites, have been attracting increasing interest because of their unique layered structure and adjustable chemical composition. The chemical formula of LDHs can be written as $\left[\mathrm{M}^{2+}{ }_{1-\mathrm{x}} \mathrm{M}^{3+}{ }_{\mathrm{x}}(\mathrm{OH})_{2}\right]^{\mathrm{x+}} \mathrm{A}_{\mathrm{x} / \mathrm{n}}{ }^{\mathrm{n}-} \cdot \mathrm{m} \mathrm{H}_{2} \mathrm{O}$, where $\mathrm{M}^{2+}$ is a divalent metal ion such as $\mathrm{Ca}^{2+}, \mathrm{Mg}^{2+}, \mathrm{Co}^{2+}, \mathrm{Fe}^{2+}, \mathrm{Cu}^{2+}$, or $\mathrm{Ni}^{2+} ; \mathrm{M}^{3+}$ is a trivalent ion such as $\mathrm{Al}^{3+}, \mathrm{Fe}^{3+}, \mathrm{Cr}^{3+}$; and $\mathrm{A}^{\mathrm{n}-}$ is the interlayer anion such as $\mathrm{NO}_{3}{ }^{-}$or $\mathrm{CO}_{3}{ }^{2-}$. The value of $\mathrm{x}$ usually lies in the range of $0.33>\mathrm{x}>0.22^{17}$. Recent research indicates that $\mathrm{LDH}$, as a synergistic fire retardant agent in polymer/IFR systems, can not only catalyze the formation of carbonaceous residue during thermal decomposition, but also enhance the thermal stability of polymer composites and make the resulting char layer more stable and compact ${ }^{18-20}$. Zhang et al. ${ }^{21}$ systematically investigated the influence of sodium dodecyl sulfate (SDS) intercalated ZnAl, MgAl, CaAl, and CuAl-LDHs on intumescent fire retardant (IFR) PP/LDH nanocomposites. The results indicate that the PP/IFR/ZnAl-LDH nanocomposite showed a significant peak heat release rate (PHRR) reduction, of around 75\%, with 1 wt\% loading of ZnAl-LDH, which demonstrates that LDHs consisting of different metal ions have a different effect on the fire retardancy of the polymer. As is known, nickel and its compounds possess excellent catalytic performance ${ }^{22-24}$. Song et al. ${ }^{25}$ investigated the synergistic effect of a supported nickel catalyst with intumescent fire-retardants in polypropylene. The results indicate that adding the nickel catalyst can significantly improve the thermal stability of PP systems. Wang et al. ${ }^{26}$ investigated the influence of different metal ions in an alginate additive, indicating that nickel alginate exhibits better fire retardancy than other ion-alginates, and nickel ions have a catalytic effect on the nickel alginate pyrolysis process, yielding incombustible products. Yang et al. ${ }^{27}$ reported that nickel salts and nickel oxide can increase LOI values in PP/IFR system.

Therefore, making use of the versatility in the composition of LDHs, nickel ions could be introduced into the layers of LDHs so as to catalyze the conversion of polymer molecules into char during combustion, then improving fire retardant efficiency. Recently, some articles report 
that when LDHs were modified by organic anions such as $\mathrm{SDBS}^{28}$, $\mathrm{DBS}^{29}, \mathrm{SPDP}^{30}, \mathrm{CEPPA}^{31}$, and SIEPDP ${ }^{32}$ etc., they greatly increased the interlayer distance and thus enhanced the dispersion properties and the fire-retardant properties of the composites. Wilen and colleagues have synthesized a series of azo-based FRs such as azo (R-N=N-R) and/or triazenes (R-N=N-N$\mathrm{R})$, which achieved excellent fire retardancy in a PP system ${ }^{33,34}$ and a PE system ${ }^{35}$. Additionally, incorporation of azobenzene groups in copolyester can significantly increase the amount of residue and thermo-oxidative stability due to its high-temperature self-crosslinking characteristics ${ }^{36}$. Moreover, the azo compounds possess a good UV absorption ability ${ }^{37}$. Based on the analysis above, the introduction of azo-based compounds into the interlayers of the LDH could be a valuable way to obtain a synergistic fire retardant effect with LDH, improve the dispersion of LDH laminates (necessary to overcome the high degree of crystallinity in PP) and endow PP with good UV protection.

In this paper, ternary nickel-containing layered double hydroxides (ZnNiAl-LDH) were synthesized by a homogeneous precipitation method. Azobenzene-4,4'-dicarboxylic acid anion intercalated LDH (Azo-LDH) was synthesized using ion exchange. Various LDHs were characterized by Fourier transform infrared spectroscopy (FTIR), scanning electron microscopy (SEM), X-ray diffraction (XRD), and thermogravimetric analysis (TGA). Afterwards, Azo-LDH as a novel synergistic agent and IFR were employed to enhance the flame retardancy of PP. The morphologies, thermal stability, and flame retardancy of various PP/IFR/LDH composites were investigated systemically by X-ray diffraction (XRD), scanning electron microscopy (SEM), energy dispersive $\mathrm{X}$-ray (EDX), thermogravimetric analysis (TGA), cone calorimetry (CC), limiting oxygen index (LOI), and the UL-94 test. The morphologies and chemical composition of the char residue after CC tests were also examined.

\section{EXPERIMENTAL SECTION}

2.1. Materials. $\mathrm{Ni}\left(\mathrm{NO}_{3}\right)_{2} \cdot 6 \mathrm{H}_{2} \mathrm{O}, \mathrm{Zn}\left(\mathrm{NO}_{3}\right)_{2} \cdot 6 \mathrm{H}_{2} \mathrm{O}, \mathrm{Al}\left(\mathrm{NO}_{3}\right)_{3} \cdot 9 \mathrm{H}_{2} \mathrm{O}, \mathrm{NaNO}_{3}, \mathrm{HNO}_{3}, \mathrm{p}-$ nitrobenzoic acid, sodium hydroxide, urea, and pentaerythritol (PER) were obtained from Sinopharm Chemical Reagent Co., Ltd. and used without further purification. Ammonium polyphosphate (APP, 203G) was manufactured by Shifang Taifeng New Flame Retardant Co., Ltd.. Polypropylene (PP, T30S) was purchased from China Petroleum and Chemical Corporation, Suzhou, Kunshan, Jiangsu Branch of Oil.

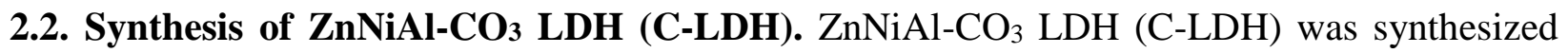
using the homogeneous precipitation method with urea as a source of hydroxyl ${ }^{38}$. In brief, $2.95 \mathrm{~g}$ $\mathrm{Ni}\left(\mathrm{NO}_{3}\right)_{2} \cdot 6 \mathrm{H}_{2} \mathrm{O}(0.01 \mathrm{~mol}), 2.97 \mathrm{~g} \mathrm{Zn}\left(\mathrm{NO}_{3}\right) \cdot 6 \mathrm{H}_{2} \mathrm{O}(0.01 \mathrm{~mol}), 3.75 \mathrm{~g} \mathrm{Al}\left(\mathrm{NO}_{3}\right)_{3} \cdot 9 \mathrm{H}_{2} \mathrm{O}(0.01 \mathrm{~mol})$, and $3.0 \mathrm{~g}$ urea $(0.05 \mathrm{~mol})$ were dissolved in $75 \mathrm{~mL}$ deionized water. The mixed solution was stirred at room temperature for 30 minutes. Then the homogeneous solution was transferred into a $200 \mathrm{~mL}$ Teflon-lined autoclave and heated to $100{ }^{\circ} \mathrm{C}$ for 24 hours. After cooling to room temperature, the final product was filtered and washed several times with distilled water until the $\mathrm{pH}$ was around 7. Finally, the pale green product was dried in a vacuum oven at $60{ }^{\circ} \mathrm{C}$ until a constant weight was achieved. This product is described as C-LDH. 
2.3. Synthesis of ZnNiAl-NO $\mathrm{NO}_{3} \mathbf{L D H}$ (N-LDH). The interlayer $\mathrm{CO}_{3}{ }^{2-}$ must be converted to $\mathrm{NO}_{3}{ }^{-}$ to ensure efficient exchange ${ }^{39}$. $\mathrm{ZnNiAl}-\mathrm{NO}_{3} \mathrm{LDH}(\mathrm{N}-\mathrm{LDH})$ was obtained by ion exchange of the as-synthesized C-LDH. In a typical experiment, $4 \mathrm{~g}$ C-LDH was transferred to a three-neck flask containing $2 \mathrm{~L}$ of an aqueous solution of $\mathrm{NaNO}_{3}(1.2 \mathrm{M})$ and $\mathrm{HNO}_{3}(0.01 \mathrm{M})$. The mixture was stirred at room temperature under a $\mathrm{N}_{2}$ atmosphere for $48 \mathrm{~h}$. The precipitated LDH slurry was filtered and washed thoroughly with distilled water then dried in an oven at $60{ }^{\circ} \mathrm{C}$ overnight and denoted N-LDH.

2.4. Synthesis of Azobenzene-4,4'-dicarboxylic acid. Azobenzene-4,4'-dicarboxylic acid (AzoDBA) was synthesized using a classical method ${ }^{40} .15 \mathrm{~g}(67.5 \mathrm{mmol}) \mathrm{p}$-nitrobenzoic acid, 50g sodium hydroxide $\left(1.25 \mathrm{~mol}\right.$ ) and $225 \mathrm{~mL} \mathrm{H}_{2} \mathrm{O}$ were placed into a $500 \mathrm{~mL}$ round-bottomed flask fitted with a magnetic stirrer, and the mixture was heated at $70{ }^{\circ} \mathrm{C}$ until the solid dissolved completely. Then, hot aqueous glucose (100 g in $150 \mathrm{~mL}$ deionized water) was added slowly, whereupon a yellow precipitate was obtained. This rapidly changed to a brown solution upon further addition of glucose. When all the glucose had been added, a stream of air was passed through the mixture for $3 \mathrm{~h}$ and a light brown precipitate was obtained. Then, this precipitate was dissolved in water and acidified with dilute acetic acid, whereupon a light pink precipitate was obtained (see Scheme 1). The resulting light pink precipitate was filtered and washed with distilled water, then dried in an oven at $60^{\circ} \mathrm{C}$ to obtain azobenzene-4,4'-dicarboxylicacid (yield 9 g, 80\%). FTIR (KBr, $\mathrm{cm}^{-1}$ ): 3467, 3095, 2979, 2928, 2667, 2550, 1687, 1604, 1580, 1496, 1426, 1295, 939, and 871. ${ }^{1} \mathrm{H}$ NMR (600MHz, DMSO-d 6 ) d (ppm): 8.07 (d, 4H), 7.87 (d, 4H) (see Figure $\mathrm{S} 1$ in the Supporting Information).
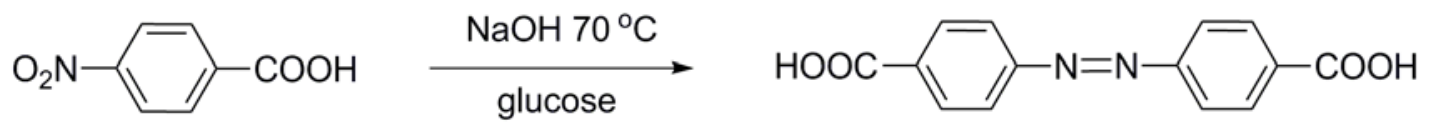

Scheme 1 The synthetic route of Azo-DBA

2.5. Synthesis of Azo-LDH (O-LDH). O-LDH was prepared by ion exchange. 1g N-LDH was transferred to a three-neck flask fitted with a magnetic stirrer. Then, $100 \mathrm{~mL}$ of a $0.1 \mathrm{M}$ aqueous solution of azobenzene-4,4'-dicarboxylic acid, previously adjusted to $\mathrm{pH} 7.5$ by using $2 \mathrm{M}$ aqueous solution of $\mathrm{NaOH}$, was also added. The mixture was stirred at room temperature under $\mathrm{N}_{2}$ for $24 \mathrm{~h}$. Finally, the pale-yellow precipitate was filtered and washed several times with distilled water, then dried in an oven at $60{ }^{\circ} \mathrm{C}$ overnight and denoted O-LDH.

2.6. Preparation of $\mathbf{P P / I F R / L D H ~ C o m p o s i t e s . ~ D r i e d ~ L D H s ~ p o w d e r s , ~ i n t u m e s c e n t ~ f l a m e ~}$ retardant (IFR), (mass ratio of APP : PER is 3:1) and polypropylene (PP) were combined in a mixer (XSS-300 torque rheometer, Rubber Machinery Co., Ltd. Shanghai Branch) at a temperature of $180^{\circ} \mathrm{C}$ with a rotor speed of $20 \mathrm{rpm}$ for $15 \mathrm{~min}$. After cooling, the mixed material was crushed by using a strong plastic crusher (PC-230, Machinery Co., Ltd. Heiken). Different test samples were prepared by injection moulding (EM80-V, Chun-Plastic Machinery Co., Ltd). The formulations of various samples are given in Table 1. Scheme 2 shows the overview of the processes involved, starting from the synthesis of O-LDH to the preparation of the PP composites. 
Table 1. Formulations and flame retardancy of various PP/IFR/LDH composites

\begin{tabular}{ccccccc}
\hline samples & PP & IFR & C-LDH & O-LDH & \multicolumn{2}{c}{ Fire retardancy } \\
\cline { 6 - 7 } & /wt\% & /wt\% & /wt\% & /wt\% & LOI $/ \%$ & UL94 \\
& & & & & & \\
\hline PP & 100 & & & & 17.0 & NR \\
PI & 75 & 25 & & & 26.3 & V-1 \\
PIOL1 & 75 & 24 & & 1 & 29.3 & V-0 \\
PIOL2 & 75 & 23 & & 2 & 28.8 & V-1 \\
PIOL3 & 75 & 22 & & 3 & 27.0 & V-1 \\
PIOL4 & 75 & 21 & & 4 & 26.0 & NR \\
PIOL5 & 75 & 20 & & 5 & 23.8 & NR \\
PIL1 & 75 & 24 & 1 & & 27.0 & V-1 \\
PIL2 & 75 & 23 & 2 & & 28.6 & V-1 \\
PIL3 & 75 & 22 & 3 & & 28.1 & V-1 \\
PIL4 & 75 & 21 & 4 & & 25.6 & NR \\
PIL5 & 75 & 20 & 5 & & 24 & NR \\
\hline
\end{tabular}

2.7. Measurements. X-ray diffractometer (XRD) patterns were collected by Rigaku Denki Co., Ltd., D / max-1400 X-ray diffractometer equipped with a $\mathrm{Cu}-\mathrm{K} \alpha$ radiation $(\lambda=1.5406 \mathrm{~nm})$ at 40 $\mathrm{kV}$ and $30 \mathrm{~mA}$. The scan range of $\mathrm{LDH}$ and O-LDH was from $3^{\circ}$ to $80^{\circ}$ and the scanning rate was $2 \%$ min. The scanning range of PP composites was from $0.5^{\circ}$ to $80^{\circ}$.

Fourier transform infrared spectra (FTIR) were obtained on a Perkin Elmer Spectrum One spectrophotometer with a scanning range from 4000 to $400 \mathrm{~cm}^{-1}$ and a resolution of $4 \mathrm{~cm}^{-1}$ using $\mathrm{KBr}$ discs.

The surface morphologies of samples were investigated using an UItra55 scanning electron microscope (SEM, Germany Carl Zeiss NTS GmbH). The LDH powders were ultrasonically dispersed in ethanol and then dropped onto a conducting copper tape. The surface morphologies of various samples, and their char residues formed after cone calorimetry, were investigated using SEM. Elemental analysis of the chars, and the dispersion properties of LDH, were investigated by scanning electron microscopy with an energy-dispersive X-ray (EDX) analyzer at an accelerating voltage of $15 \mathrm{kV}$. All samples were sputter coated with a thin platinum layer to improve the conductivity before examination.

Thermogravimetric analyses (TGA) were carried out using a TGA/SDTA 851e thermal analyzer. All samples were heated from $30{ }^{\circ} \mathrm{C}$ to $800{ }^{\circ} \mathrm{C}$ at a constant heating rate of $10{ }^{\circ} \mathrm{C} / \mathrm{min}$ under air, with a flow rate of $50 \mathrm{~mL} / \mathrm{min}$.

The flammability of the composites was investigated using a cone calorimeter (Fire Testing Technology, UK) according to ISO 5600. All samples had dimensions of $100 \times 100 \times 4 \mathrm{~mm}^{3}$ and were irradiated horizontally at a heat flux of $35 \mathrm{~kW} \cdot \mathrm{m}^{-2}$. The measurements were done in triplicate and the average data are reported. The limiting oxygen index (LOI) was measured according to ASTM D 2863-97 with test strips of dimensions $130 \times 6.5 \times 3.2 \mathrm{~mm}^{3}$. The UL-94 
vertical burning test was performed in a test chamber (Fire Testing Technology, UK), and the specimens for testing were of dimensions $130 \times 13 \times 3 \mathrm{~mm}^{3}$. The formulations, LOI, and UL-94 test data are summarized in Table 1.

UV transmission spectra were collected using a UV-3150 spectrometer, using samples compressed into thin pellets.

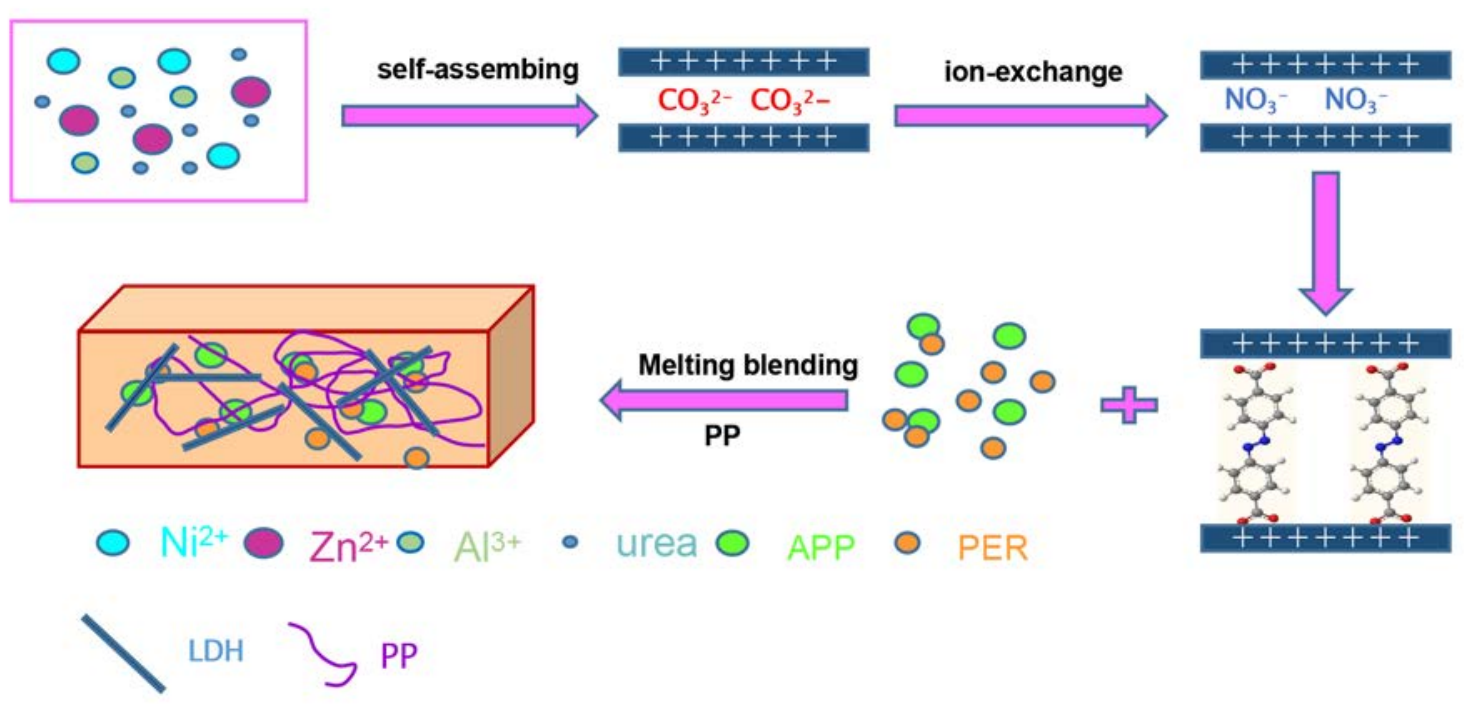

Scheme 2 Diagrammatic illustration of the synthetic route of Azo-DBA Modified LDH and preparation process of $\mathrm{PP} / \mathrm{IFR} / \mathrm{LDH}$ composites

\section{RESULTS AND DISCUSSION}

\subsection{Structural and Morphologic Characterization of Various LDHs.}

XRD diffraction patterns of C-LDH, N-LDH and azo-modified LDH (O-LDH) are displayed in Figure 1. The XRD pattern of C-LDH clearly reveals that C-LDH is highly crystalline and monodispersive. Its first basal reflection (003) appearing at $2 \theta=11.40^{\circ}$ corresponds to an interlayer distance of $0.78 \mathrm{~nm}$. With regard to N-LDH, the (003) reflection shifts to $2 \theta=9.84^{\circ}$ indicating an interlayer distance of $0.89 \mathrm{~nm}$. The typical characteristic peaks of C-LDH completely disappear, and a new series of intense basal reflections at lower angles appear indicating the complete replacement of $\mathrm{CO}_{3}{ }^{2-}$ by $\mathrm{NO}_{3}{ }^{-}$, which is in good agreement with the previous literature ${ }^{41}$. As for O-LDH, the position of (003) reflection shifted to $2 \theta=4.14^{\circ}$ indicating an interlayer distance of $2.10 \mathrm{~nm}$, suggesting that azobenzene-4,4'-dicarboxylic acid anions had been successfully intercalated into the interlayers of N-LDH. Moreover, no residual peaks of N-LDH remain, demonstrating a highly efficient intercalation. The SEM images of various LDH particles are shown in Figure 2. The pristine C-LDH particles (Figure 2a and b) 
possess a typical disk-like geometrical structure, the average diameter of the C-LDH lamellae is 1-2 $\mu \mathrm{m}$ and the thickness is about 30-40 $\mathrm{nm}$. The existence of irregular edges on most of the particles suggests the incomplete process of crystal growth, leading to no particular particle shape. The morphological features of the O-LDH particles (Figure 2c and d) are similar to CLDH and still maintain the typical disk-like structure.

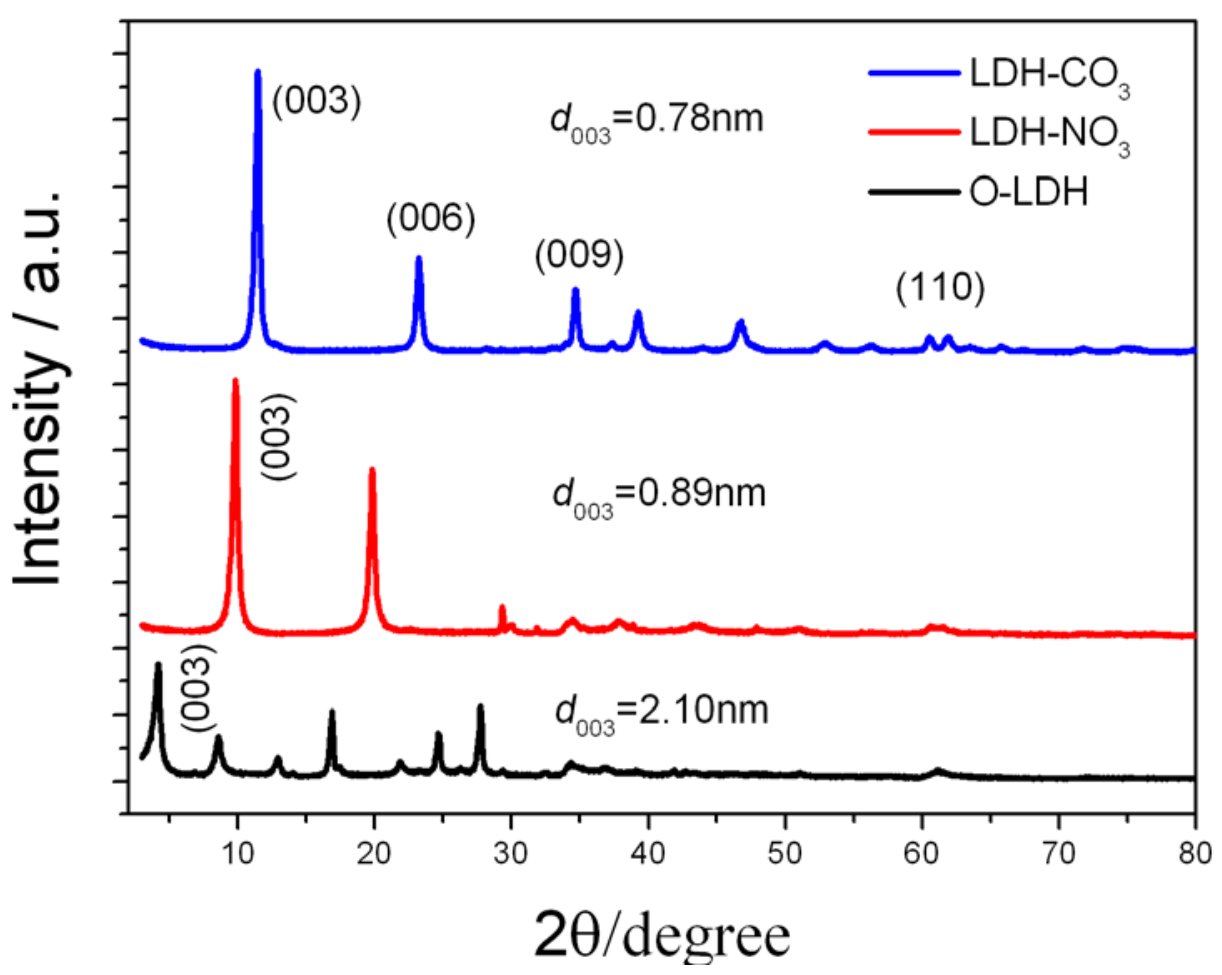

Figure 1 XRD patterns of C-LDH, N-LDH and O-LDH 

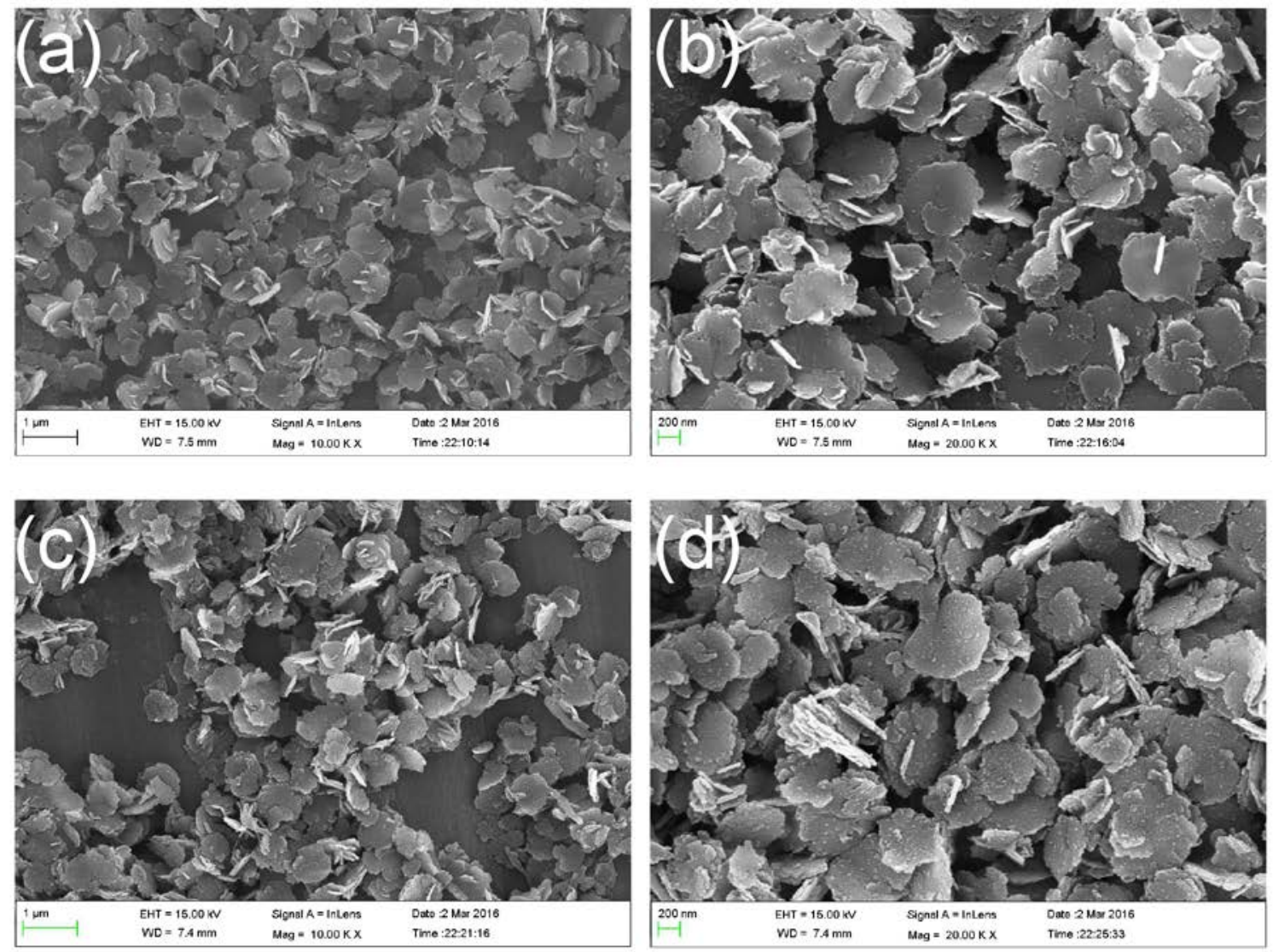

Figure 2 SEM images of the C-LDH $(\mathrm{a}, \mathrm{b})$ and O-LDH $(\mathrm{c}, \mathrm{d})$

\subsection{Fourier Transform Infrared Spectra (FTIR).}

FTIR spectra of C-LDH, N-LDH, O-LDH and azobenzene-4,4'-dicarboxylic acid (Azo-DBA) are presented in Figure 3. Figure 3 (a) and (b) show the FTIR spectra of C-LDH and N-LDH respectively. A broad absorption band at around 3466 and $3434 \mathrm{~cm}^{-1}$ originates from the stretching vibration of -OH groups of the metal hydroxide layer and interlayer water in C-LDH and N-LDH. For C-LDH and N-LDH, the bending vibration of the interlayer water is also reflected in the peaks at 1626 and $1631 \mathrm{~cm}^{-1}$, respectively ${ }^{42}$. The characteristic band for interlayer carbonate and nitrate can be observed at 1384 and $1380 \mathrm{~cm}^{-1}$, respectively ${ }^{43}$. The disappearance of the peak of carbonate and the appearance of the peak of nitrate suggest that the 


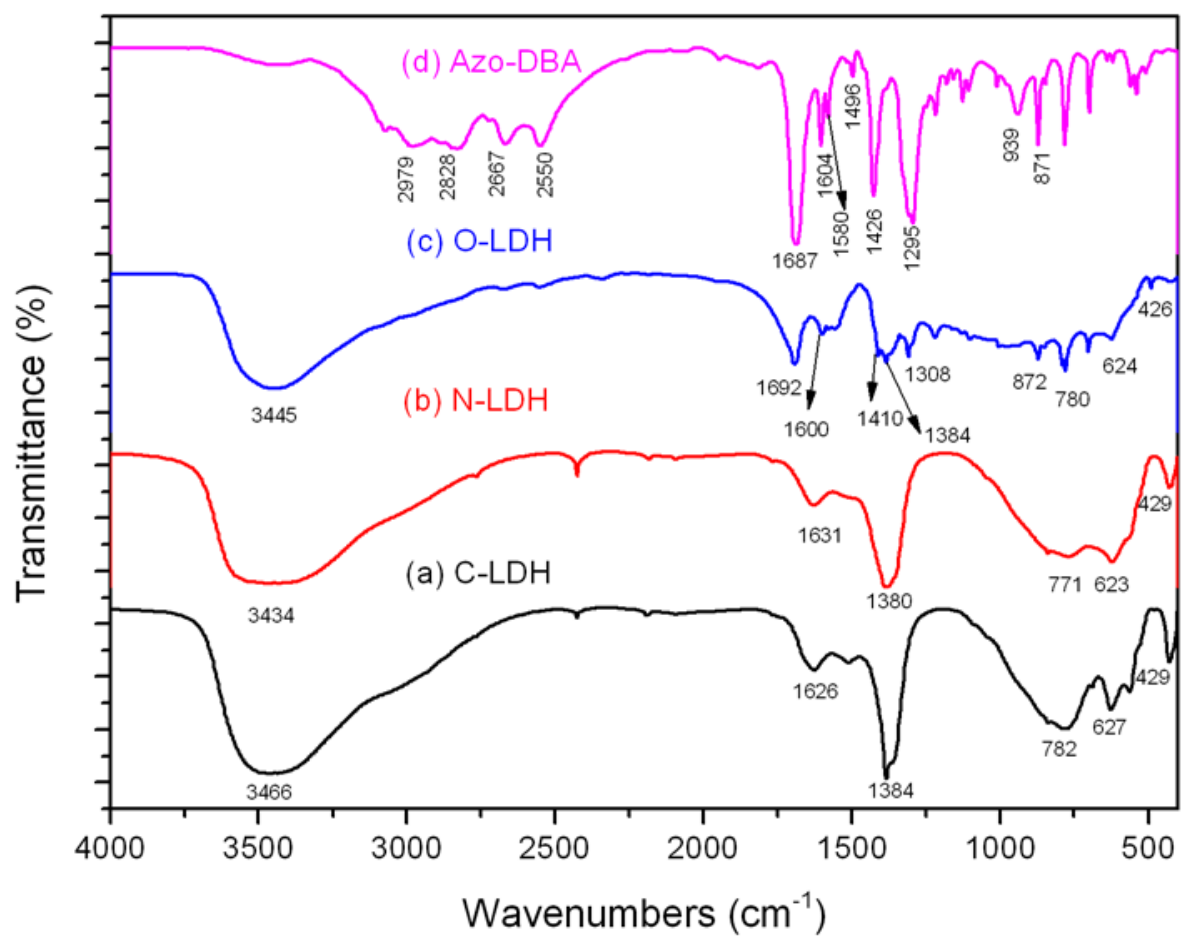

Figure 3 FTIR spectra of C-LDH (a), N-LDH (b), O-LDH (c) and Azo-DBA (d)

carbonate has been completely exchanged by nitrate, which is consistent with XRD analysis. For all the LDH samples, the bands recorded below $800 \mathrm{~cm}^{-1}$ can be attributed to the vibrations of metal oxides (M-O, O-M-O). In the spectrum of Azo-DBA, a broad and strong peak at 2550$3200 \mathrm{~cm}^{-1}$ can be assigned to the -COOH groups, and the strong peak at $1687 \mathrm{~cm}^{-1}$ is associated with the $\mathrm{C}=\mathrm{O}$ vibration ${ }^{44}$. The characteristic peaks at 1580 and $1496 \mathrm{~cm}^{-1}$ can be attributed to the vibration of the benzene ring. The peak at $1604 \mathrm{~cm}^{-1}$ should be assigned to the asymmetric stretching vibration of $\mathrm{N}=\mathrm{N}^{45}$. The characteristic peak at $1295 \mathrm{~cm}^{-1}$ belongs to the aromaticnitrogen bond ${ }^{46}$. All characteristic peaks of azo-DBA appear in the spectrum of O-LDH. Taken in combination with the result of XRD analysis, it can be concluded that azo-DBA molecules have been intercalated into the interlayer space of N-LDH. In addition, the characteristic absorption band of interlayer nitrates almost completely disappears in the case of O-LDH, indicating that the nitrates have been almost completely replaced by the Azo-DBA anion.

\subsection{Structural Characterization of PP/IFR/LDH Composites.}

XRD patterns of PP and its composites are shown in Figure 4. Figure 4a shows the basic reflections of PP at $2 \theta=14.30^{\circ}, 14.14^{\circ}, 17.12^{\circ}, 18.95^{\circ}$ and $21.31^{\circ}$ corresponding to the monoclinic $\alpha$ form. Figure $4 \mathrm{~b}$ exhibits the XRD patterns of sample PI (PP/IFR). As can be seen, the introduction of IFR does not change the polymorphism of PP. With respect to PIL1 and PIOL1 composites (Figure 4c and d), no change in the polymorphism of PP is observed in the 
presence of C-LDH and O-LDH platelets either. Moreover, no visible characteristic diffraction peaks of O-LDH are retained in the XRD patterns of PIOL1 and PIL1 composites, suggesting that highly exfoliated and/or well-intercalated LDH platelets exist in the PP matrix, or there is too little LDH content. To further investigate the dispersion of the LDH particles, the SEM and SEM-EDX element mapping were carried out. The results of SEM and SEM-EDX are presented in Figure 5. In detail, the $\mathrm{P}$ signal, in red, represents the IFR; the $\mathrm{C}$ signal, in white, is a representation of the polypropylene chains and the $\mathrm{Al}$ signal, in green, represents the C-LDH or O-LDH particles. As shown in Figure 5a, no obvious aggregation appearance can be observed, implying a homogeneous dispersion of IFR particles and O-LDH layers in the PP matrix. This result can be confirmed by the results of SEM-EDX, as can be seen from Figure 5a1-a3, the SEM-EDX element mappings of $\mathrm{P}, \mathrm{C}$, and $\mathrm{Al}$ signal display no elemental signal enrichment, suggesting that IFR and O-LDH could be well-distributed and dispersed in the PP matrix. However, the SEM image of PIL1 composites (Figure 5b) reveals that the dispersion of IFR and C-LDH in the PP matrix is poor. The EDX element mapping of PIL1 (Figure 5b1) reveals that carbon is unevenly distributed. The grouping of $\mathrm{Al}$ or $\mathrm{P}$ element signals also implies that the $\mathrm{C}$ LDH and IFR particles aggregate. Research has shown that the IFR particles are preferentially attracted to the LDH surfaces, which increases their dispersion in the polymer matrix ${ }^{47}$. So, if the LDH aggregates, the IFR will accordingly aggregate, in agreement with the result of SEM-EDX mapping. Based on previous analysis, the conclusion can be drawn that the organic modification could improve the dispersion of LDH in PP matrix due to the greater interlayer distance, making it easier for the PP molecular chain to intercalate between the layers.

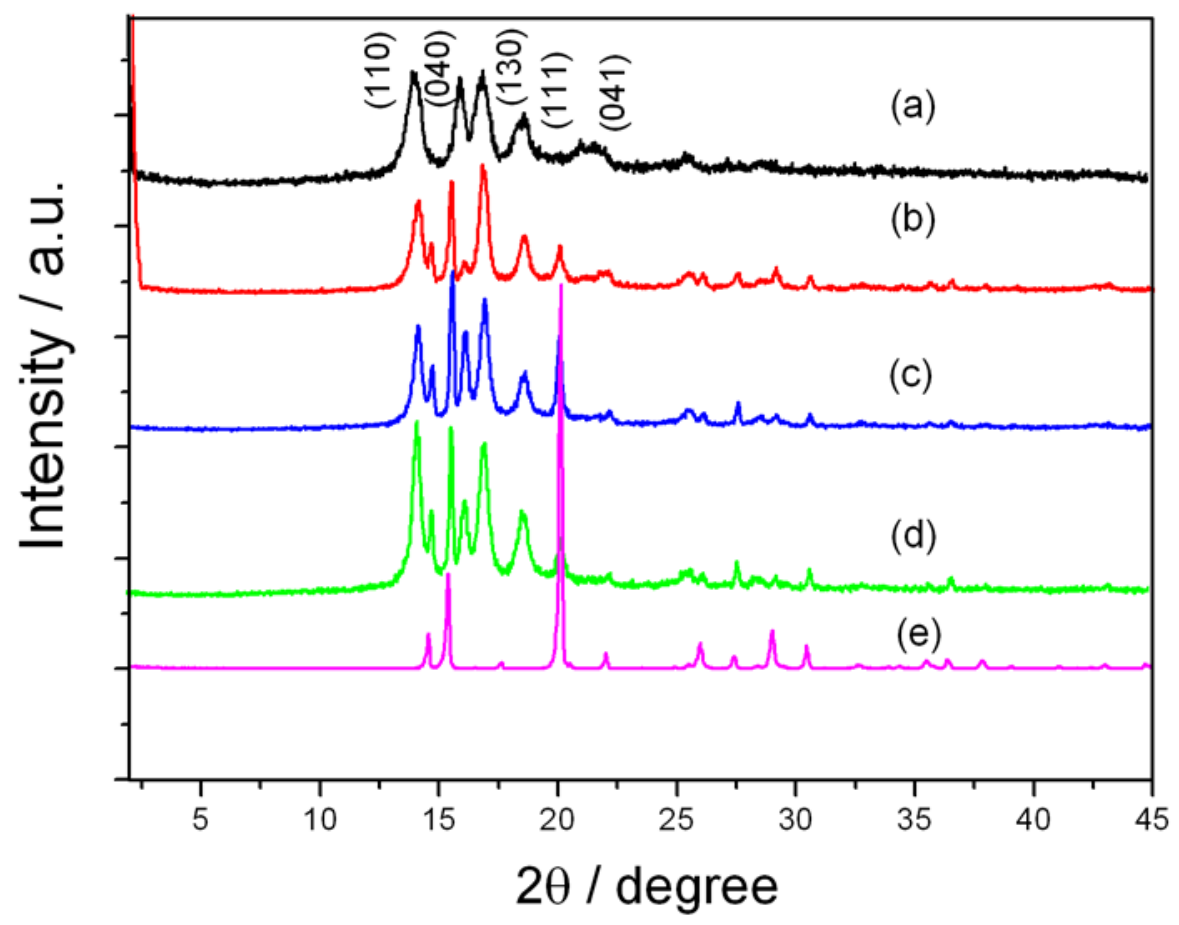

Figure 4 XRD patterns of PP(a), PI(b), PIL1(c), PIOL1(d), and IFR(e) 

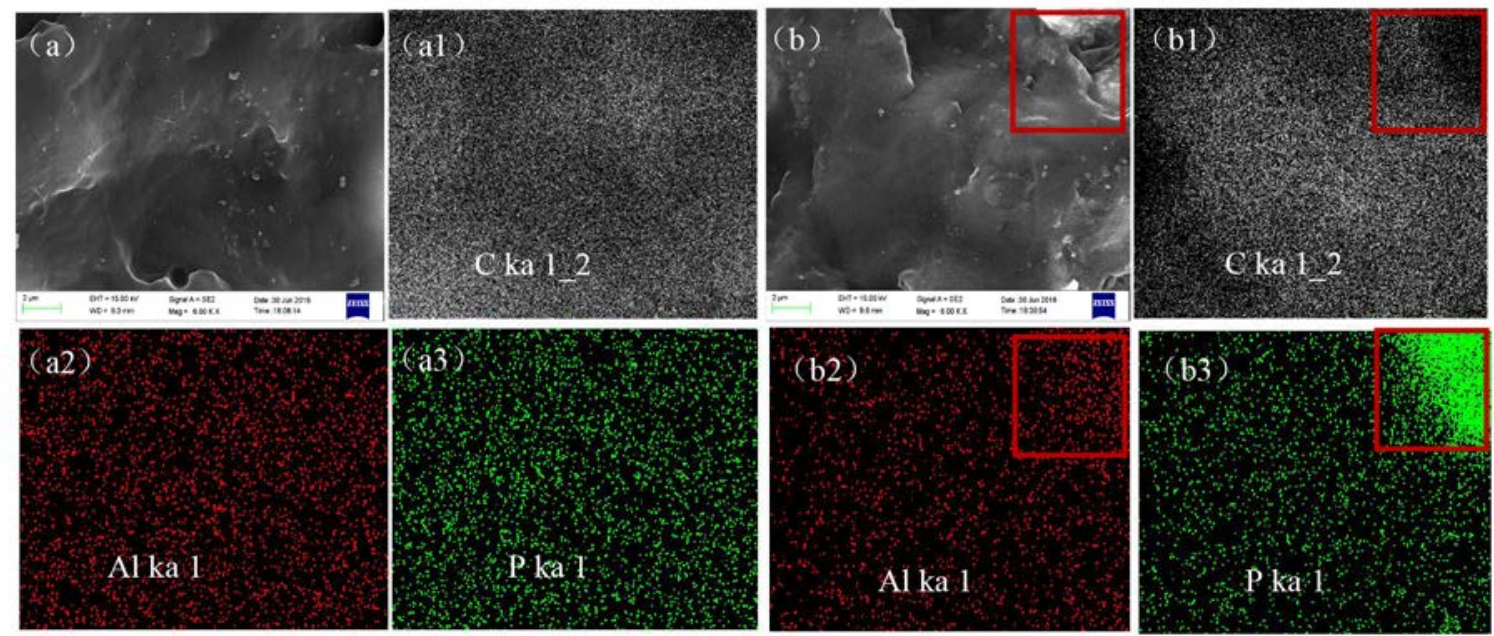

Figure 5 SEM images and EDX elements mapping images ( $\mathrm{C}$ in white, $\mathrm{P}$ in green and $\mathrm{Al}$ in red) of PIOL1 (a-a4) and PIL1 (b-b4) composites

\subsection{Thermal Stability of C-LDH, O-LDH and PP/IFR/LDH Composites.}

3.4.1 Thermal degradation behavior of C-LDH and O-LDH. The TGA curves for C-LDH and O-LDH in air are presented in Figure 6. As can be seen, the C-LDH shows two main thermal decomposition steps. The first decomposition step occurs between 100 and $200{ }^{\circ} \mathrm{C}$, and may be due to the loss of the physically absorbed and interlayer water molecules of the C-LDH. The second step is from 250 to $500{ }^{\circ} \mathrm{C}$, which is attributed to the dehydroxylation of the LDH layers and the loss of carbonate ${ }^{48}$. For O-LDH, the thermal degradation behavior of O-LDH can be divided into three stages. The first stage, below $200{ }^{\circ} \mathrm{C}$, is due to the loss of adsorbed and interlayer water. The second stage, from 250 to $420^{\circ} \mathrm{C}$, can be attributed to the thermal decomposition of Azo-DBA chain in the LDH layers. The third stage occurs between 430 and $600{ }^{\circ} \mathrm{C}$ and may be due to the dehydroxylation of the $\mathrm{LDH}$ layers. It is worth noting that the temperature of the dehydroxylation of the LDH layers increases, which can be attributed to the hindrance effect of char residue resulting from the decomposition of the Azo-DBA chain, indicating that introducing the Azo-DBA anion as an intercalator into the interlayer can increase the interlayer space, and improve the thermal stability of $\mathrm{LDH}$, to some extent ${ }^{49}$. The char residues of C-LDH and $\mathrm{O}-\mathrm{LDH}$ at $700{ }^{\circ} \mathrm{C}$ are $66.9 \%$ and $39 \%$, respectively. This result is further evidence for the successful organo-modification of LDH via the ion exchange route. 


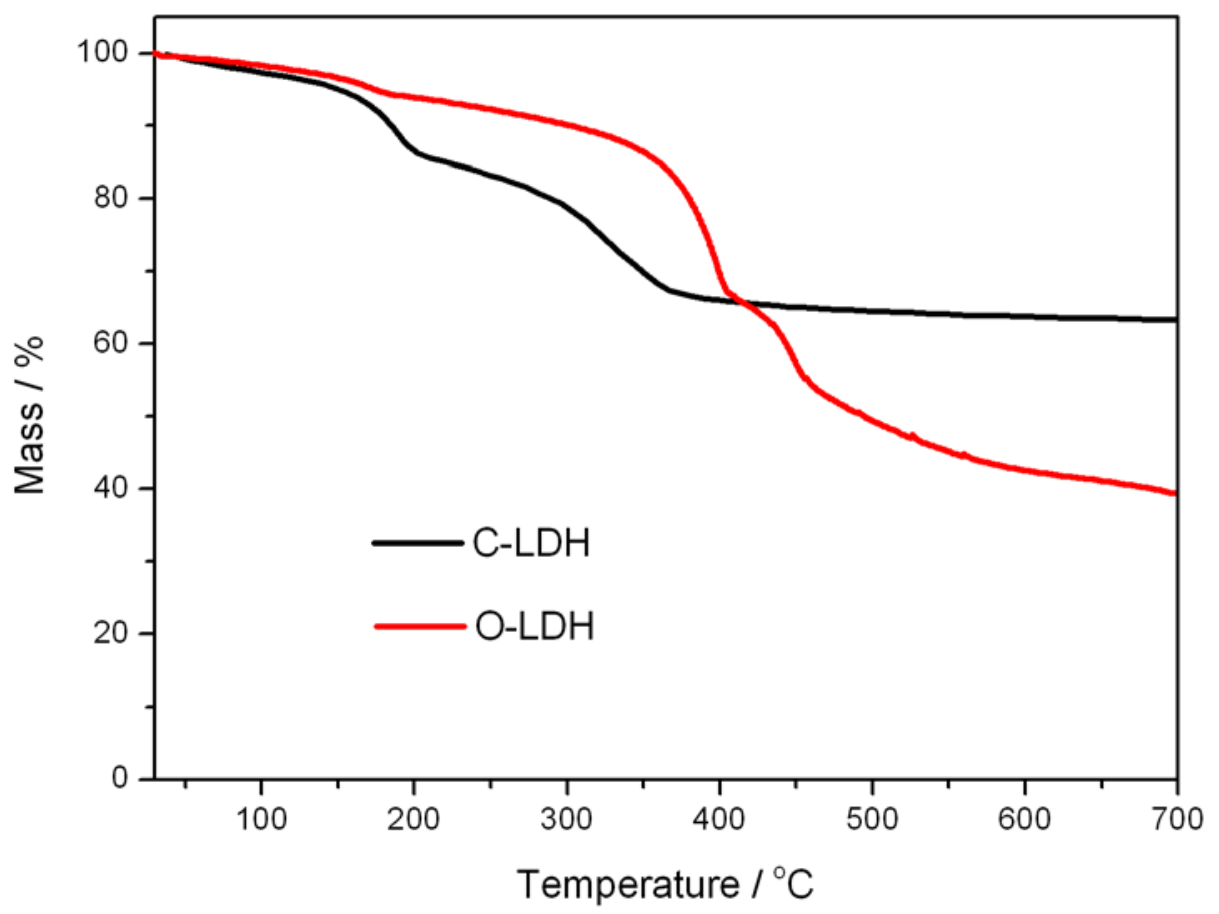

Figure 6 TGA curves for C-LDH and O-LDH under an air atmosphere

\subsubsection{Thermal Degradation Behavior of $P P$ and its Composites.}

The TGA curves for neat PP and its composites under air atmosphere are shown in Figure 7 and the detailed data are presented in Table 2. In general, the thermal stability is evaluated by $T_{\text {onset }}$ (the temperature at $5 \%$ mass loss), $T_{-50 \%}$ (the temperature of $50 \%$ mass loss) and the resulting char residual percentage. According to Table 2, $T_{\text {onset, }} T_{-50 \%}$ and the char yield for all PP composites are higher than that of pure PP. As can be seen in Figure 7, pure PP shows a single thermal decomposition stage between 250 and $400{ }^{\circ} \mathrm{C}$, corresponding to a $T-50 \%$ at $329{ }^{\circ} \mathrm{C}$ and almost no residue is left at a higher temperature. Compared with neat PP, the PI composite shows two main thermal decomposition steps and exhibits obvious increases in $T_{\text {onset }}$ and $T_{-50 \%}$ (about 30 and $58{ }^{\circ} \mathrm{C}$, respectively), and the char residue is $4 \%$ at $800{ }^{\circ} \mathrm{C}$. 


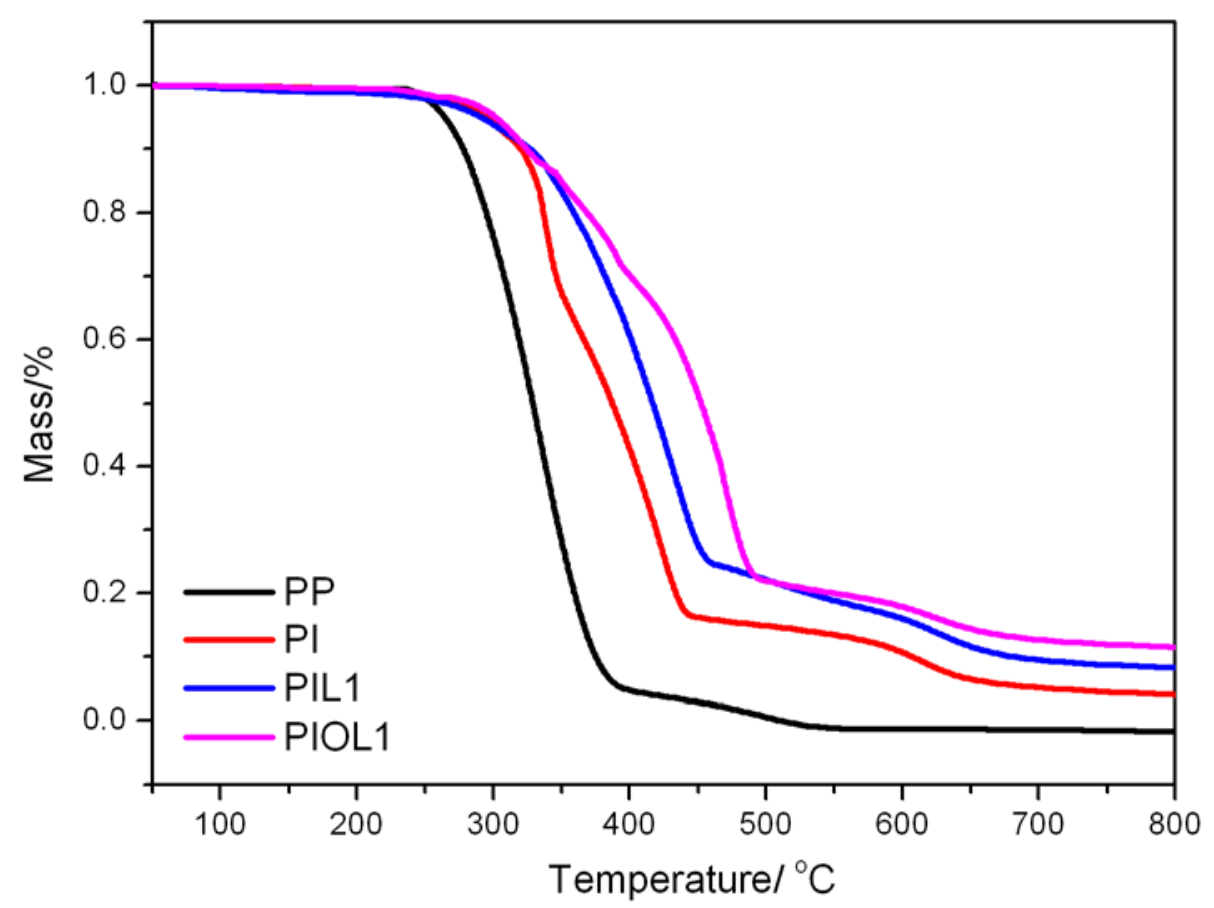

Figure 7 TGA curves for PP, PI, PIL1 and PIOL1 under air atmosphere

Table 2 Thermal analysis data of PP and its composites at air atmosphere

\begin{tabular}{cccc}
\hline samples & $T_{\text {onset }} /{ }^{\circ} \mathrm{C}$ & $T_{-50 \%} /{ }^{\circ} \mathrm{C}$ & $\begin{array}{c}\text { Char yield } \\
\text { at } 800{ }^{\circ} \mathrm{C} / \mathrm{wt} \%\end{array}$ \\
\hline PP & 264 & 329 & 0 \\
PI & 294 & 387 & 4 \\
PIL1 & 271 & 397 & 8.2 \\
PIOL1 & 282 & 432 & 11.4 \\
\hline
\end{tabular}


The results can be attributed to the thermal decomposition of IFR, generating phosphoric and polyphosphoric acids, which will promote the formation of the char layer through dehydrogenation, hinder the transfer of heat and oxygen, and thus protect the underlying matrix from being consumed ${ }^{50}$. In comparison with PI, PIL1, the TGA curve of PIOL1 shifts to the higher temperature. The value of $T_{-50 \%}$ is increased owing to the barrier effect of LDH layers and the dilution by water released from $\mathrm{LDH}{ }^{51}$. Moreover, the char yield at $800{ }^{\circ} \mathrm{C}(8.2 \%)$ is insreased, due to the formation of more stable and continuous char layer by the catalytic function of metal ions in C-LDH. In contrast to the PIL1 composite, the PIOL1 composite exhibits greater thermal stability. The $T_{\text {onset }}$ and $T_{-50 \%}$ of the PIOL1 composites are significantly increased by 11 and $35{ }^{\circ} \mathrm{C}$, respectively, which can be attributed to better dispersion and compatibility of O-LDH than the original C-LDH in PP. Similar results have also been observed in other polymer / nanofiller composites ${ }^{52}$. However, the PIL1 and PIOL1 composites exhibit a slight reduction in $T_{\text {onset, }}$ which is due to the metal ions of LDH layers $(\mathrm{Ni}, \mathrm{Zn})$, which could catalyze the earlier thermal degradation of the PP chain and IFR system, so that the early charring would be beneficial to the preservation of underlying PP matrix. It is clear that the simultaneous addition of IFR and O-LDH can largely enhance the char yield, $11.4 \%$ at $800{ }^{\circ} \mathrm{C}$, suggesting an optimum synergistic effect between O-LDH and IFR.

\subsection{Flammability Characterization of $P P$ and its Composites.}

3.5.1. Burning Behaviors (LOI and UL-94 Tests.). The LOI value and UL-94 ratings of pure PP and its composites are summarized in Table 1 . As can be seen, neat PP exhibits an LOI value of $17 \%$ and is not classified in the UL-94 vertical test. When $25 \mathrm{wt} \%$ of IFR is added, the LOI value is increased to $26.3 \%$ with a UL-94 V-1 rating. When $1 \mathrm{wt} \%$ of IFR is replaced by C-LDH, the LOI value of PIF1 goes a little up to $27 \%$ with a UL-94 V-1 rating. However, the PIOL1 formulation exhibits a higher LOI value of $29.3 \%$ and achieves the UL-94 V-0 rating. This reduction in flammability is believed to result from: the better dispersion of O-LDH particles in PP matrix; the radical-trapping effect of the azo group; and the synergistic effect between OLDH and IFR. Thus it will lead to a more stable and compact char layer, which could prevent the pyrolysis and combustion of the underlying PP matrix. Increasing the amount of C-LDH from 2 $\mathrm{wt} \%$, or O-LDH from $1 \mathrm{wt} \%$, the LOI values significantly decrease, presumably because the higher loading of LDH leads to agglomeration. In summary, there is an optimum loading ratio of LDH and IFR for the best synergistic fire-retardant effect in the PP composites, corresponding to the ratio giving the maximum char yield in TGA.

\subsubsection{Cone Calorimeter Test.}

The combustion behavior of pure PP and its IFR composites have been investigated using a cone calorimeter. The important parameters, including time to ignition (TTI), peak heat release rate (PHRR), time to peak heat release rate (TTPHRR), total heat release (THR), and fire growth rate index (FIGRA) $)^{53}$, are summarized in Table 3. The measured HRR curves for pure PP and its composites are presented in Figure 8(a). It can be observed that pure PP burns rapidly after ignition, showing a sharp peak with a PHRR value of $720 \mathrm{~kW} \mathrm{~m}^{-2}$. In contrast to neat PP, the 
PHRR values of PI, PIL1, and PIOL1 are 267, 296, and $271 \mathrm{~kW} \mathrm{~m}^{-2}$, respectively (approximately $62.9 \%, 58.9 \%, 62.3 \%$ reductions), showing that the addition of IFR and LDH greatly reduces the PHRR of PP composites. The HRR curves and PHRR values of PIOL1 and PIL1 show slightly greater HRRs than the PI composite. However, compared with PI composites, the TTPHRR values were delayed $10 \mathrm{~s}$ and $35 \mathrm{~s}$ for PIL1 and PIOL1, respectively. Based on Table 3,

Table 3 Cone calorimeter data of PP and its composites

\begin{tabular}{ccccccc}
\hline Samples & TTI & TTPHRR & PHRR & THR & Residue & FIGRA \\
& $/ \mathrm{s}$ & $/ \mathrm{s}$ & $/ \mathrm{kW} \mathrm{m}^{-2}$ & $\mathrm{MJ} \mathrm{m}^{-2}$ & $/ \%$ & $/ \mathrm{kW} \mathrm{m}^{-2} \mathrm{~s}^{-1}$ \\
\hline PP & 50 & 170 & 720 & 136 & 8.9 & 4.2 \\
PI & 39 & 250 & 267 & 111 & 23.9 & 1.1 \\
PIL1 & 35 & 260 & 296 & 109 & 25.0 & 1.1 \\
PIOL1 & 39 & 285 & 271 & 102 & 30.7 & 1.0 \\
\hline
\end{tabular}

pure PP has released a total heat release of $136 \mathrm{MJ} \mathrm{m}^{-2}$. As expected, incorporating $25 \mathrm{wt} \%$ IFR into PP decreases the THR to $111 \mathrm{MJ} \mathrm{m}^{-2}$, an $18.4 \%$ reduction. Simultaneous addition of IFR and LDH can further reduce THR: it can be seen that PIL1 and PIOL1 show a decline of 19.8\% and $25 \%$, respectively. This reduction may result from the catalytic charring function of LDH, which can lead to more PP chains participating in the carbonization process, and therefore, less volatile products forming "fuel" to go into the combustion zone ${ }^{52}$. It is worth noting that incorporating $1 \mathrm{wt} \%$ O-LDH can result in better fire retardancy compared with C-LDH, which may be caused by the improved dispersion of the O-LDH in PP matrix. The fire growth rate index (FIGRA) is a parameter defined as the ratio of PHRR to time to PHRR, which can be used to evaluate the fire growth of a material. All the PP composites show decreased FIGRA compared to that of pure PP. The most striking result is observed in the case of the PIOL1 composite, showing a 78\% reduction in FIGRA relative to pure PP. Total smoke production (TSP) curves of PP and its composites are shown in Figure 8(b). The TSP values of PP and its composites samples are 64.5, 34.8, 29.1, and $26.3 \mathrm{~m}^{2}$, respectively, for pure PP, PI, PIL1, and PIOL1. The TSP shows that the incorporation of IFR significantly reduces the TSP and $1 \mathrm{wt} \%$ loading of LDH further decreases the TSP of PIL1 and PIOL1, indicating an obvious smoke suppression benefit between LDH and IFR, and showing signs of more complete combustion ${ }^{54}$. Notably, PIOL1 still shows a better smoke suppression than PIL1. As shown in Table 3, the PIOL1 system displays the highest char residue after CC tests (approximately $30.7 \%$ ). From the results of CC tests, the PP composite with 1 wt\% O-LDH and 24 wt\% IFR has lower flammability than that containing $25 \mathrm{wt} \%$ IFR. 

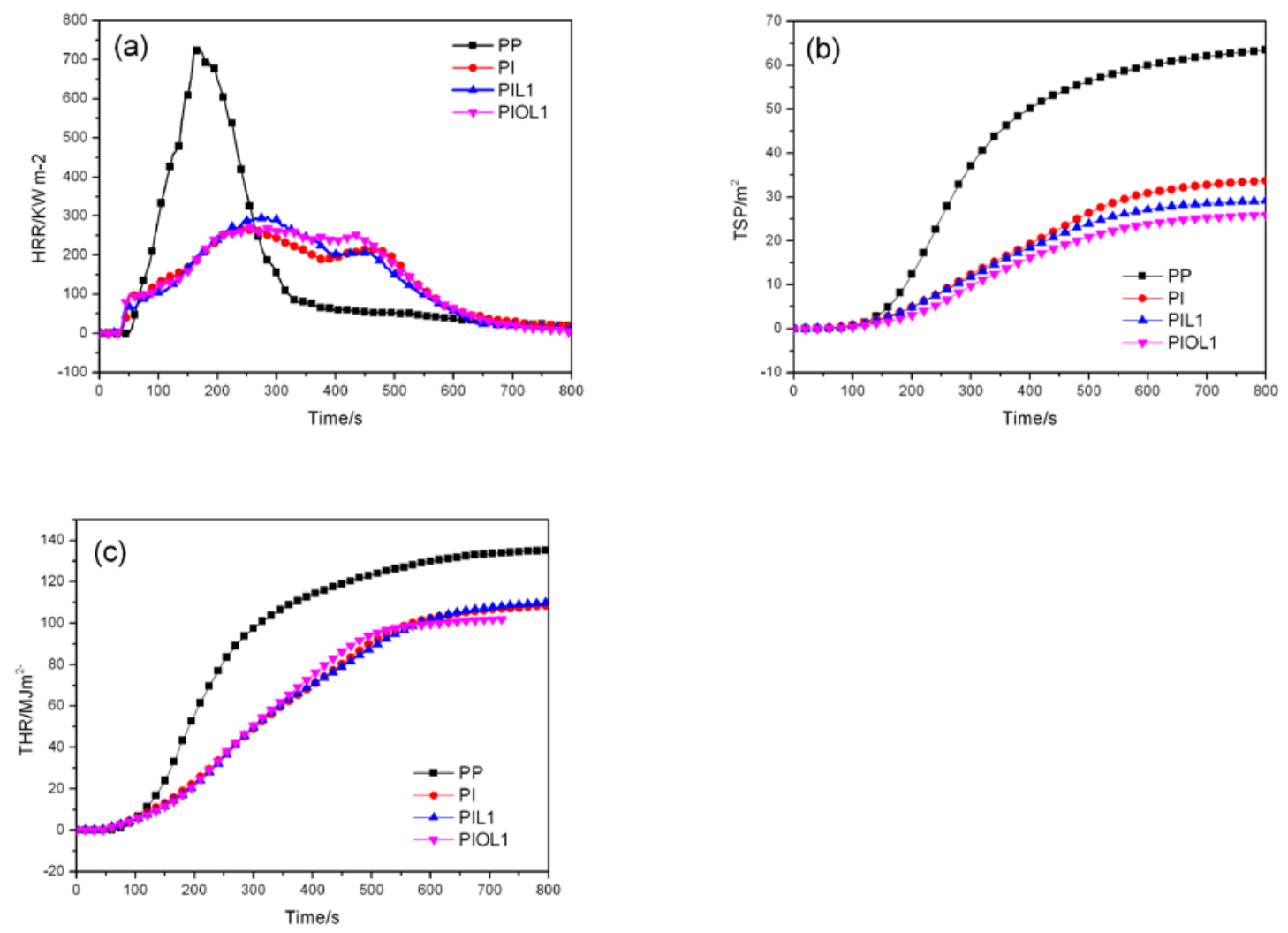

Figure 8 HRR (a), TSP (b), and THR (c) plots of PP and its composites

\subsection{Flame Retardant Mechanism.}

Photographs of the residual char after CC testing are shown in Figure 9 (a, b, c, d). There is almost no residue left for pure PP. All the composites containing IFR exhibit typical intumescent charring; the char of PIOL1 is very compact and strong, while the char of PI and PIL1 are more fragile and cracked. The microstructure of residual char was further investigated by SEM and the representative results are shown in Figure 9 (a1, b1, c1). The char of PI has a rough and porous 

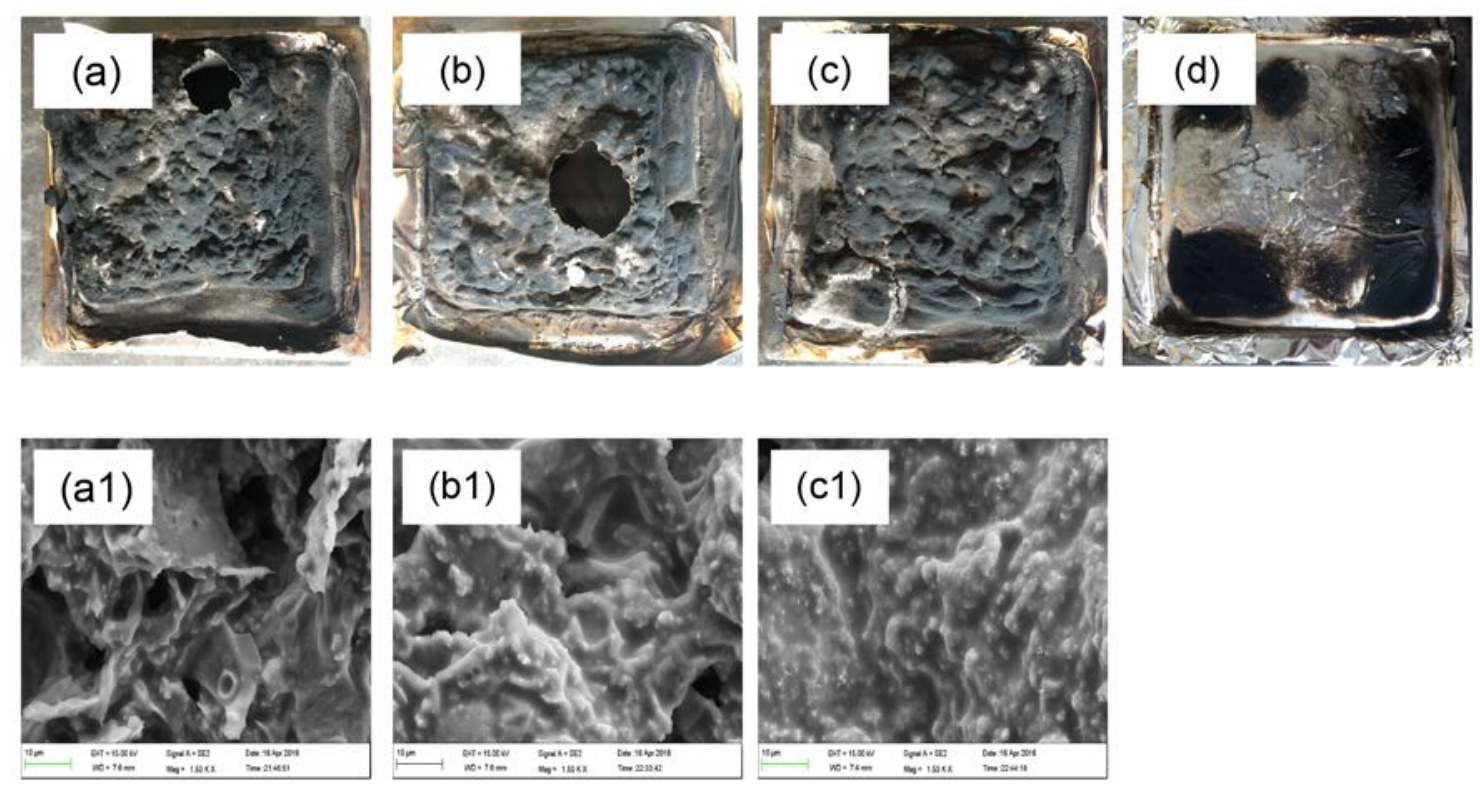

Figure 9 Digital photographs and SEM images of burning residues of PI (a) (a1), PIL1 (b) (b1), PIOL1 (c) (c1) and PP (d) after CC

surface which provides less protection for underlying PP matrix. The PIL1 composite takes on a smooth and dense char layer, but a few cracks still exist; finally, the char layer of PIOL1 possesses a more compact and continuous surface without cracks, indicating that O-LDH can prevent the collapse of the char layer and play an important role in the process of dense charformation, owing to its improved dispersion in the PP matrix. This dense and continuous char layer can prevent fuel volatilization and hinder oxygen and heat feedback during burning of PP, resulting in a higher char residue and better fire retardant properties of PIOL1. In general, during combustion, LDH lamellae may migrate from the interior of the sample toward the surface due to the numerous rising bubbles of thermal degradation products and the associated convection flow in the melt, which will increase the density of the char ${ }^{55}$. For example, the elemental compositions of $\mathrm{Ni}$ and $\mathrm{Zn}$ in the char surface after CC tests are listed in Figure 11. It can be seen that there are dramatically higher concentrations of Ni and Zn for PIOL1 than that of PIL1, which demonstrate the migration of O-LDH over the C-LDH, improving the char promotion function of $\mathrm{Ni}^{2+}$ and $\mathrm{Zn}^{2+}$. Moreover, the azo-modifier can also provide nitrogen and low energy radicals during thermolysis ${ }^{33}$. These more stable radicals may reduce the concentration of high energy $\mathrm{H} \cdot, \cdot \mathrm{OH}$ and $\mathrm{O} \cdot$ radicals and quench the flame; nitrogen may also contribute to fire retardancy by diluting the flammable gases ${ }^{56}$. The production rates of $\mathrm{CO}$ and $\mathrm{CO}_{2}$ from cone calorimetry are shown in Figure 10a and Figure 10b, respectively. PIOL1 shows a higher CO and a lower $\mathrm{CO}_{2}$ production rate compared to PIL1, indicating that the low energy radicals originated from the azo-modifier have replaced the higher energy $\mathrm{H} \cdot, \cdot \mathrm{OH}$ and $\mathrm{O} \cdot$ radicals, therefore resulting in flame quenching, or flame retardancy through incomplete combustion. This phenomenon demonstrates the gas phase flame retarding effect of azo-modifier. 

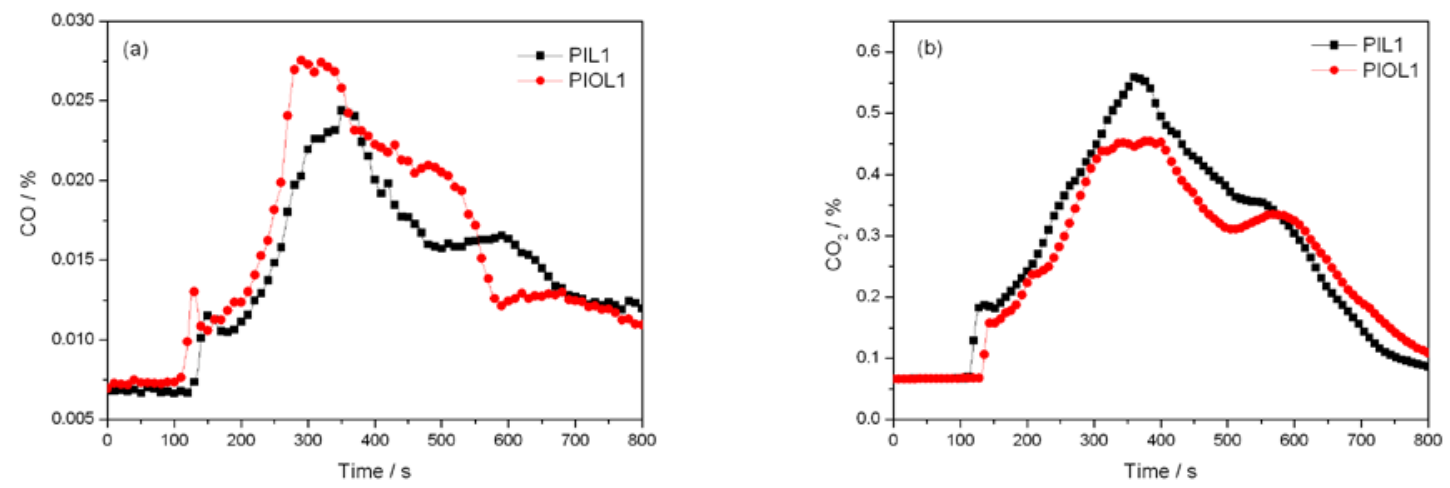

Figure $10 \mathrm{CO}$ (a) and $\mathrm{CO}_{2}$ (b) product rate curves of PIL1 and PIOL1 as a function of time in CC tests
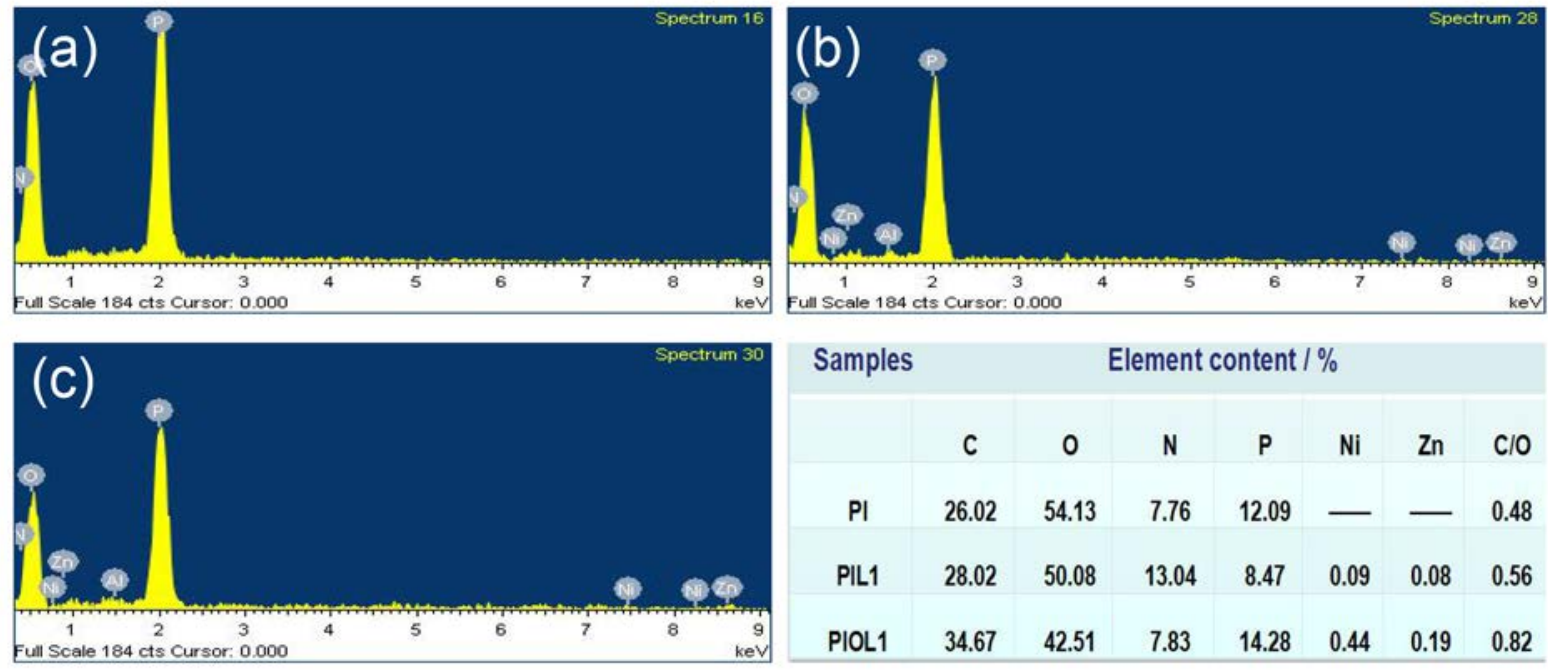

Figure 11 EDX spectra of residue of PI (a), PIL1 (b), and PIOL1 (c) after CC tests

Based on the analysis above, it can be concluded that O-LDH has a greater effect with IFR than C-LDH. Figure 11 presents the results of the elemental analysis for the residue of PI, PIL1, and PIOL1 after CC testing. As shown in Figure 11, the carbon content of 26, 28, and 34 wt\% was found in the residue of PI, PIL1, and PIOL1, respectively, indicating that the addition of LDH improves the carbonization ability of $\mathrm{PP} / \mathrm{IFR}$ composites. The $\mathrm{C} / \mathrm{O}$ ratio in the residual char of PIOL1 is 0.82, which is dramatically higher than that of PI (0.48) and PIL1 (0.56) composites, suggesting that the incorporation of $\mathrm{O}-\mathrm{LDH}$ can improve the thermal oxidative-resistance of the char layer and retain much more carbon. Depending upon the aforementioned analysis, the proposed flame retardant mechanism is illustrated in Scheme 3. There is no doubt that the denser, continuous char layer of PIOL1 with high thermal-resistance is more effective in restraining the spread of the flame and oxygen, protecting the underlying polymer matrix of PIOL1. 


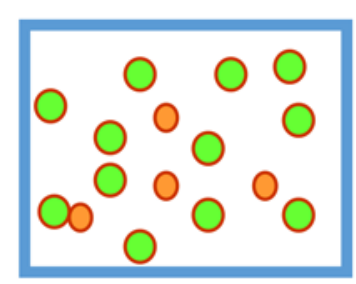

$\mathrm{PI}$

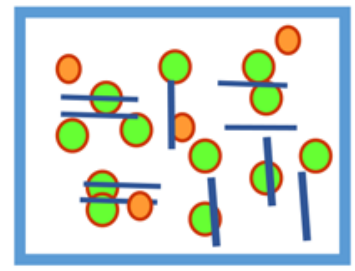

PIL 1

Agglomerated

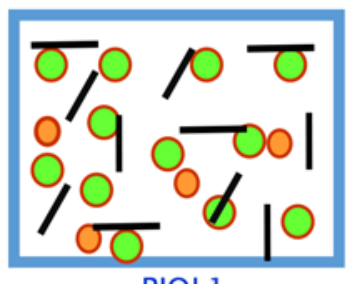

PIOL1

Well dispersed
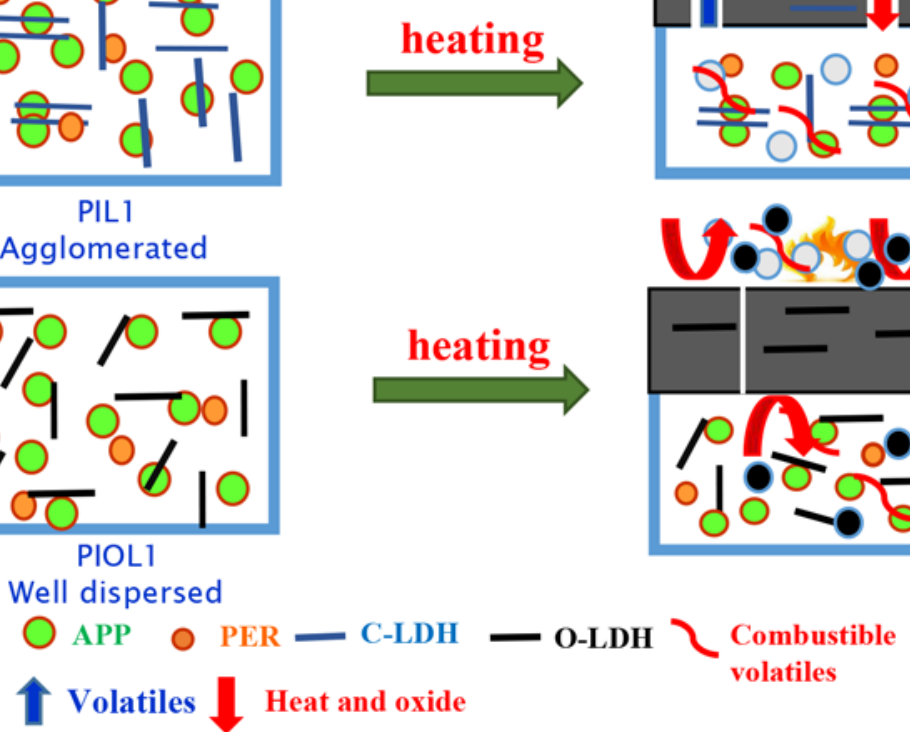
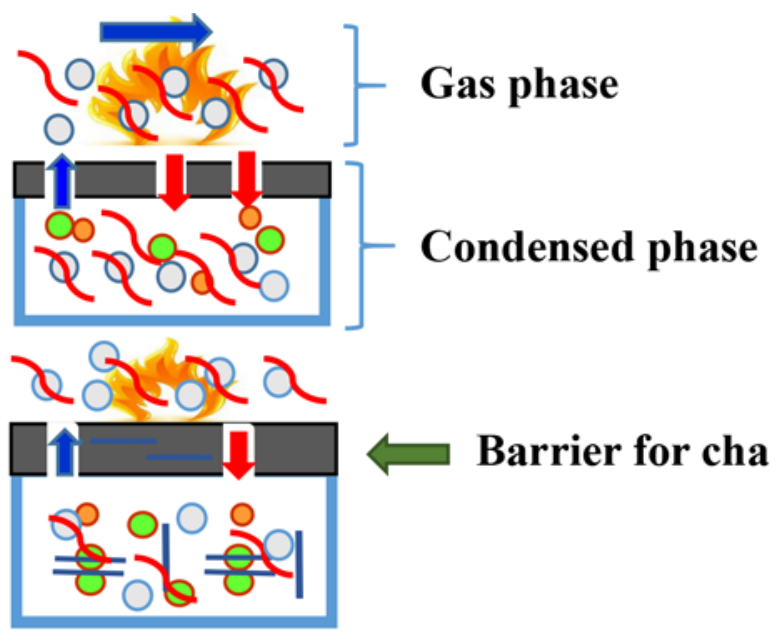

$\Leftarrow$ Barrier for char

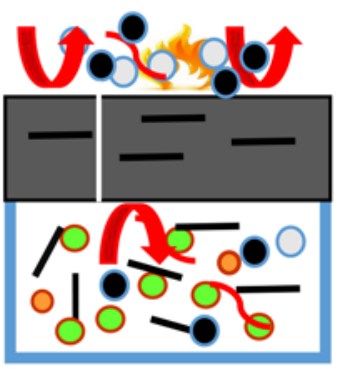

Barrier for char

(1)

High energy radicals

Scheme 3 Diagrammatic illustration of the flame retardant mechanism of PP composites

\subsection{Mechanical Properties.}

The mechanical properties of fire retardant PP composites are also an important consideration for practical usability. Typical tensile stress versus strain curves for PP and its composites are shown in Figure 12a. Figure 12b displays the Young's modulus and the ultimate tensile strength. These show that the neat PP exhibits a tensile strength of $34.37 \mathrm{MPa}$ and a Young's modulus of 4.60 MPa. Compared with neat PP, the PI composite shows a reduction in tensile strength, Young's modulus, and elongation at break. In general, increased additive loading is likely to cause deterioration in mechanical properties of the polymer matrix, due mainly to poor additive-matrix compatibility ${ }^{57}$. 

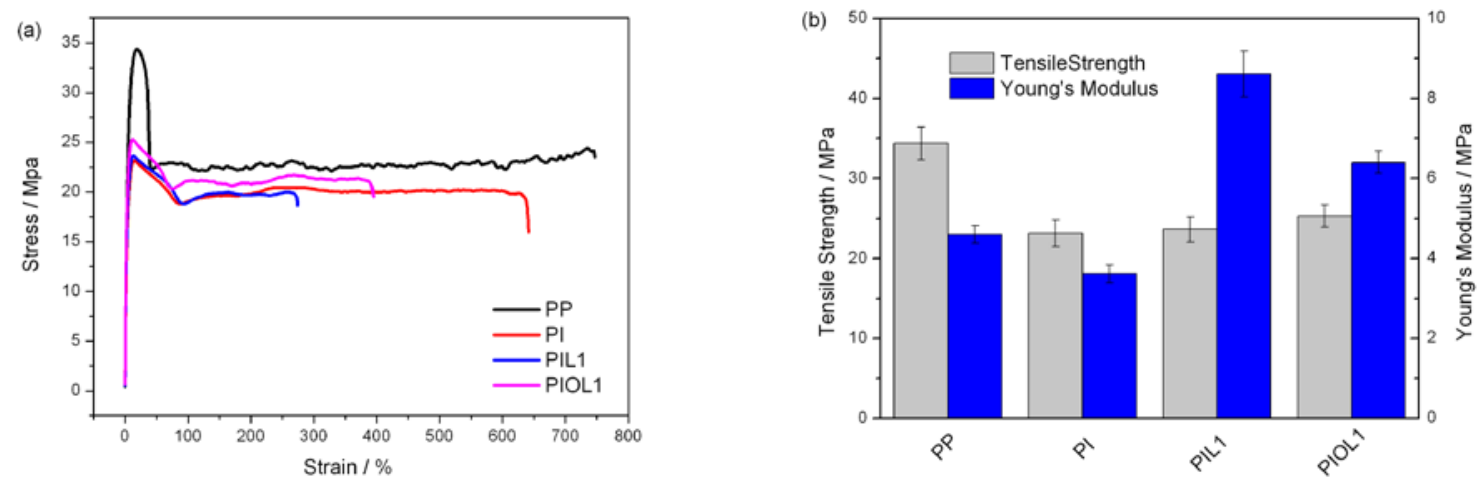

Figure 12 Stress-strain curves of PP and its composites (a), ultimate tensile strength and Young's modulus (b) of the PP and its composites

When 1 wt\% of IFR is replaced by C-LDH, the tensile strength is slightly increased and the Young's modulus is improved by $58 \%$ to $8.61 \mathrm{MPa}$, due to reinforcement from the LDH. However, the elongation at break of PIL1 composites is significantly decreased (a $57 \%$ reduction) compared with PI composites. This probably results from poor compatibility of CLDH particles with the PP matrix, while their uneven dispersion will lead to stress concentration. On the other hand, there was a noticeable improvement in both tensile strength and elongation at break for PIOL1 compared with PIL1, which can be attributed to the organic modification of C$\mathrm{LDH}$, then improving the compatibility between the PP matrix and O-LDH particles. The improvement in the elongation at break and tensile strength indicates that the O-LDH also functions as a strengthening agent, reinforcing the PP composite matrix.

\subsection{UV Protection.}

The effect of O-LDH on UV protection performance of fire retardant PP composites was also studied. It is well known that azo-compounds have good UV resistance. The UV resistance capacity of PP the composites was evaluated by measuring the UV absorbance spectrum and the results are shown in Figure 13. It can be seen that the O-LDH shows strong UV absorption 


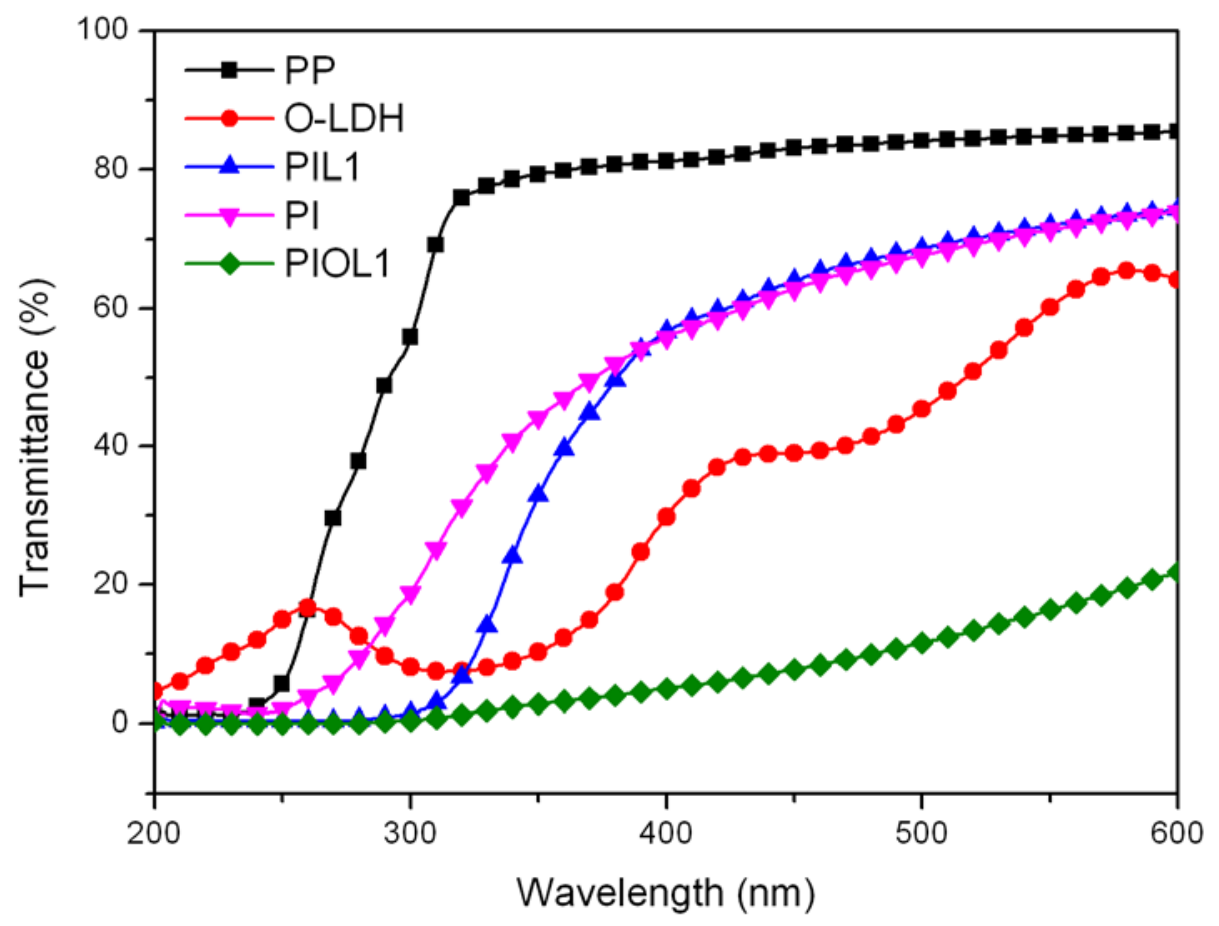

Figure 13 UV transmission curves of PP, O-LDH and its composites

between 280 and $450 \mathrm{~nm}$, which results from the presence of azo-DBA anions in the interlayer galleries of LDH. The UV absorbance of neat PP indicates a weak UV absorption capacity, particularly in the range of 300-600 $\mathrm{nm}$. All PP composites show a lower UV transmission intensity than that of the pure PP. In contrast to PI composite, adding the C-LDH shows no obvious improvement in the UV protection ability. However, when C-LDH replaced by O-LDH, the UV transmission intensity of PIOL1 shows a significant reduction by $73 \%$ compared with PP, because the Azo-DBA anions serve as a UV absorber. This result shows that $1 \mathrm{wt} \%$ O-LDH provides significant UV protection for PP composites

\section{CONCLUSION}

In this work, the azo-DBA anion intercalated ternary nickel-containing layered double hydroxide (O-LDH) has been successfully synthesized using ion exchange. The structure, morphology and thermal properties were confirmed by FTIR, SEM, XRD, and TGA. Intumescent flame retardant PP composites based on IFR and LDH were prepared by melt blending. The XRD, SEM and EDX elements mapping observations of PP composites confirm that O-LDH exhibits better dispersion than C-LDH, which may be favored by the physical interaction during melt blending between O-LDH and the IFR particles. The PIOL1 displays the higher thermal stability ( $\mathrm{T}_{-50 \%}$ increases almost $103{ }^{\circ} \mathrm{C}$ compared to neat PP) compared to PI and PIL1. Thus ternary nickelcontaining layered double hydroxides really enhance the flame retardancy of PP/IFR systems and 
O-LDH exhibits a better fire retardancy with IFR than C-LDH. TGA results show that the presence of $\mathrm{C}-\mathrm{LDH}$ or $\mathrm{O}-\mathrm{LDH}$ can promote char formation. In particular, for O-LDH, the addition of 1 wt\% O-LDH can further improve the thermal stability and the char yield of PP composites. The addition of $1 \mathrm{wt} \% \mathrm{O}-\mathrm{LDH}$ results in a reduction in the total heat release (THR) values and a greater reduction in the smoke production of the PP/IFR system. The reduction in $\mathrm{CO}_{2}$ and increase in $\mathrm{CO}$ production rate in cone calorimetry provides clear evidence of the dual role played by the azo-modification: in the condensed phase it enhances the dispersion of the $\mathrm{LDH}$; in the gas phase it replaces the high energy $\mathrm{H} \cdot, \cdot \mathrm{OH}$ and $\mathrm{O} \cdot$ radicals, so acting as a condensed phase fire retardant, and a gas phase flame retardant. Moreover, the incorporation of LDH can improve the Young's modulus and tensile strength compared to using IFR alone. UV absorption shows that introducing $1 \mathrm{wt} \% \mathrm{O}-\mathrm{LDH}$ can significantly enhance UV protection of the PP/IFR system. In all, we have demonstrated that the thermal stability, mechanical property, flame resistance and UV absorption ability of PP composites can be enhanced by adding only 1 wt\% O-LDH. This procedure could be employed for the fabrication of high-performance flame retardant and anti-ultraviolet aging polymer composites.

\section{ASSOCIATED CONTENT}

\section{Supporting Information}

The Supporting Information is available free of charge on the ACS Publications website at DOI: 10.1021/ acs. iecr. 6b03953

The 1H-NMR of Azo-DBA (PDF)

\section{AUTHOR INFORMATION}

Corresponding Author: Xiaoping Hu, E-mail to: huxiaoping@swust.edu.cn

Corresponding Author: T. Richard Hull, E-mail to: trhull@uclan.ac.uk

\section{Author Contributions}

The manuscript was written through contributions of all authors. All authors have given approval to the final version of the manuscript.

The authors declare no competing financial interest.

\section{ACKNOWLEDGMENTS}

This paper was financially supported by the National Natural Science Foundation of China (51373140; 51673160) and the China Scholarship Council (201508515104).

\section{ABBREVIATIONS}

FTIR, Fourier transform infrared spectroscopy; XRD, X-ray diffractometer; SEM, scanning electron microscopy; TGA thermogravimetric analysis; EDX, energy dispersive X-ray; LOI, limit oxygen index; CONE, cone calorimeter; UV, ultraviolet; NiZnAl-LDH, nickel-containing 
layered double hydroxide; LDH, layered double hydroxide; Azo-DBA, azobenzene-4,4'dicarboxylic acid; PP, polypropylene; IFR, intumescent flame retardants; PER, pentaerythritol; APP, Ammonium polyphosphate; C-LDH, ZnNiAl-CO $\mathrm{CO}_{3} \mathrm{LH}$; N-LDH, ZnNiAl-NO 3 LDH; OLDH, Azo-ZnNiAl LDH; PI, 75wt\% polypropylene / 25wt\% intumescent flame retardant; PIL1, $75 \mathrm{wt} \%$ polypropylene / 24wt\% intumescent flame retardant / 1wt\% ZnNiAl-CO 3 LDH; PIOL1, $75 \mathrm{wt} \%$ polypropylene / 24wt\% intumescent flame retardant / 1wt\% Azo-ZnNiAl LDH.

\section{REFERENCES}

(1) Zhang, X.; Yan, X. R.; He, Q. L.; Wei, H. G.; Long, J.; Guo, J.; Gu, H. B.; Yu, J. F.; Liu, J. J.; Ding, D. W.; Sun, L. Y.; Wei, S. Y.; Guo, Z. H. Electrically Conductive Polypropylene Nanocomposites with Negative Permittivity at Low Carbon Nanotube Loading Levels. ACS Appl. Mat. Interfaces. 2015, 7, 6125-6138.

(2) Paisoonsin, S.; Pornsunthorntawee, O.; Rujiravanit, R. Preparation and characterization of ZnO-deposited DBD plasma-treated PP packaging film with antibacterial activities. Appl. Clay Sci. 2013, 273, 824-835.

(3) Morgan, A. B.; Wilkie, C. A. Flame retardant polymer nanocomposites. John Wiley \& Sons: 2007.

(4) Molyneux, S.; Stec, A. A.; Hull, T. R. The effect of gas phase flame retardants on fire effluent toxicity. Polym. Degrad. Stab. 2014, 106, 36-46.

(5) Hull, T. R.; Witkowski, A.; Hollingbery, L., Fire retardant action of mineral fillers. Polym. Degrad. Stab. 2011, 96, 1462-1469.

(6) Liang, J. Z.; Zhang, Y. J. A study of the flame-retardant properties of polypropylene/Al $(\mathrm{OH})_{3} / \mathrm{Mg}(\mathrm{OH})_{2}$ composites. Polym. Int. 2010, 59, 539-542.

(7) Li, B.; Xu, M. J. Effect of a novel charring-foaming agent on flame retardancy and thermal degradation of intumescent flame retardant polypropylene. Polym. Degrad. Stab. 2006, 91, 1380-1386.

(8) Wu, Z.; Yang, L.; Zhan, J.; Chen, Y.; Zhou, X.; Hu, Y.; Stec, A. A.; Hull, T. R. Experimental study on polystyrene with intumescent flame retardants from different scale experiments. Fire. Mater. 2016, 40, 18-26.

(9) Rimez, B.; Rahier, H.; Biesemans, M.; Bourbigot, S.; Van Mele, B. Flame retardancy and degradation mechanism of poly (vinyl acetate) in combination with intumescent flame retardants: I. Ammonium poly (phosphate). Polym. Degrad. Stab. 2015, 121, 321330

(10) Wang, D. Y.; Liu, Y.; Ge, X. G.; Wang, Y. Z.; Stec, A.; Biswas, B.; Hull, T. R.; Price, D. Effect of metal chelates on the ignition and early flaming behaviour of intumescent fireretarded polyethylene systems. Polym. Degrad. Stab. 2008, 93, 1024-1030.

(11) Wang, D.Y.; Liu, Y.; Wang, Y. Z.; Artiles, C. P.; Hull, T. R.; Price, D. Fire retardancy of a reactively extruded intumescent flame retardant polyethylene system enhanced by metal chelates. Polym. Degrad. Stab. 2007, 92, 1592-1598. 
(12) Zhang, Y.; Li, X. N.; Cao, Z. H.; Fang, Z. P.; Hull, T. R., Stec, A. A. Synthesis of zinc phosphonated poly(ethylene imine) and its fire-retardant effect in low-density polyethylene. Ind. Eng. Chem. Res. 2015, 54, 3247-3256.

(13) Wang, Q.; Undrell, J. P.; Gao, Y. S.; Cai, G. P.; Buffet, J. C.; Wilkie, C. A.; O’Hare, D. Synthesis of flame-retardant polypropylene/LDH-borate nanocomposites. Macromolecules. 2013, 46, 6145-6150.

(14) Tang, Y.; Hu, Y.; Li, B. G.; Liu, L.; Wang, Z. Z.; Chen, Z. Y.; Fan, W. C. Polypropylene/montmorillonite nanocomposites and intumescent, flame-retardant montmorillonite synergism in polypropylene nanocomposites. J. Polym. Sci., Part A: Polym. Chem. 2004, 42, 6163-6173.

(15) Vargas, A. F.; Orozco, V. H.; Rault, F.; Giraud, S.; Devaux, E.; López, B. L. Influence of fiber-like nanofillers on the rheological, mechanical, thermal and fire properties of polypropylene: an application to multifilament yarn. Compos Part A: Appl Sci Manufac. 2010, 41, 1797-1806.

(16) Du, B. X.; Fang, Z. P. Effects of carbon nanotubes on the thermal stability and flame retardancy of intumescent flame-retarded polypropylene. Polym. Degrad. Stab. 2011, 96, 1725-1731.

(17) Manzi-Nshuti, C.; Songtipya, P.; Manias, E.; Jimenez-Gasco, M. M.; Hossenlopp, J. M.; Wilkie, C. A. Polymer nanocomposites using zinc aluminum and magnesium aluminum oleate layered double hydroxides: effects of LDH divalent metals on dispersion, thermal, mechanical and fire performance in various polymers. Polymer. 2009, 50, 3564-3574.

(18) Wang, X.; Spörer, Y.; Leuteritz, A.; Kuehnert, I.; Wagenknecht, U.; Heinrich, G.; Wang, D. Y. Comparative study of the synergistic effect of binary and ternary LDH with intumescent flame retardant on the properties of polypropylene composites. RSC Adv. 2015, 5, 78979-78985.

(19) Nyambo, C.; Kandare, E.; Wilkie, C. A. Thermal stability and flammability characteristics of ethylene vinyl acetate (EVA) composites blended with a phenyl phosphonate-intercalated layered double hydroxide (LDH), melamine polyphosphate and/or boric acid. Polym. Degrad. Stab. 2009, 94, 513-520.

(20) Zhao, C. X.; Liu, Y.; Wang, D. Y.; Wang, D. L.; Wang, Y. Z. Synergistic effect of ammonium polyphosphate and layered double hydroxide on flame retardant properties of poly(vinyl alcohol). Polym. Degrad. Stab. 2008, 93, 1323-1331.

(21) Zhang, M.; Ding, P.; Qu, B. J. Flammable, thermal, and mechanical properties of intumescent flame retardant PP/LDH nanocomposites with different divalent cations. Polym. Compos. 2009, 30, 1000-1006.

(22) Lizzio, A. A.; Radovic, L. R. Transient kinetics study of catalytic char gasification in carbon dioxide. Ind. Eng. Chem. Res. 1991, 30, 1735-1744.

(23) Herring, A. M.; Mckinnon, J. T.; Mccloskey, B. D.; Filley, J.; Gneshin, K. W.; Pavelka, R. A.; Kleebe, H. J.; Aldrich, D. J. A novel method for the templated synthesis of 
homogeneous samples of hollow carbon nanospheres from cellulose chars. J. Am. Chem. Soc. 2003, 125, 9916-9917.

(24) Wen, X.; Gong, J.; Yu, H. O.; Liu, Z.; Wan, D.; Liu, J.; Jiang, Z. W.; Tang, T. Catalyzing carbonization of poly(L-lactide) by nanosized carbon black combined with $\mathrm{Ni}_{2} \mathrm{O}_{3}$ for improving flame retardancy. J. Mater. Chem. 2012, 22, 19974-19980.

(25) Song, R. J.; Zhang, B. Y.; Huang, B. T.; Tang, T. Synergistic effect of supported nickel catalyst with intumescent flame -netbatlentsabrstfllaritityrefardancy polypropylene. J. Appl. Polym. Sci. 2006, 102, 5988-5993.

(26) Liu, Y.; Zhao, J. C.; Zhang, C. J.; Guo, Y.; Cui, L.; Zhu, P.; Wang, D. Y. Bio-based nickel alginate and copper alginate films with excellent flame retardancy: preparation, flammability and thermal degradation behavior. RSC Adv. 2015, 5, 64125-64137.

(27) Wu, N.; Ding, C.; Yang, R. J. Effects of zinc and nickel salts in intumescent flameretardant polypropylene. Polym. Degrad. Stab. 2010, 95, 2589-2595.

(28) Zhang, P.; Wang, T. Q.; Qian, G. R.; Frost, R. L. Organo-LDH synthesized via tricalcium aluminate hydration in the present of Na-dodecylbenzenesulfate aqueous solution and subsequent investigated by near-infrared and mid-infrared. Spectrochim. Acta, Part A. 2014, 125, 195-200.

(29) Coiai, S.; Passaglia, E.; A. H.; Augier, S.; Pratelli, D.; Streller, R. C. The influence of the compatibilizer on the morphology and thermal properties of polypropylene-layered double hydroxide composites. Polym. Compos. 2010, 31, 744-754.

(30) Cai, J.; Heng, H. M.; Hu, X. P.; Xu, Q. K.; Miao, F. A Facile Method for the Preparation of Novel Fire-Retardant Layered Double Hydroxide and Its Application as Nanofiller in UP. Polym. Degrad. Stab. 2016, 126, 47-57.

(31) Peng, D.; Bai, K.; Jin, Z.; Yang, J. W.; Na, S.; Tang, S. F.; Shi, L. Y. Phosphoruscontaining flame retardant modified layered double hydroxides and their applications on polylactide film with good transparency. J. Colloid Interface Sci. 2015, 440, 46-52.

(32) Li, C.; Wan, J. T.; Kalali, E. N.; Fan, H.; Wang, D, Y. Synthesis and characterization of functional eugenol derivative based layered double hydroxide and its use as a nanoflame-retardant in epoxy resin. J. Mater. Chem. A. 2015, 3, 3471-3479.

(33) Aubert, M.; Nicolas, R. C.; Pawelec, W.; Wilén, C. E.; Roth, M.; Pfaendner, R. Azoalkanes-novel flame retardants and their structure property relationship. Polym. Adv. Technol. 2011, 22, 1529-1538.

(34) Pawelec, W.; Aubert, M.; Pfaendner, R.; Hoppe, H.; Wilén, C. E. Triazene compounds as a novel and effective class of flame retardants for polypropylene. Polym. Degrad. Stab. 2012, 97, 948-954.

(35) Pawelec, W.; Tirri, T.; Aubert, M.; Häggblom, E.; Lehikoinen, T.; Skåtar, R.; Pfaendner, R.; Wilén, C.-E. Toward halogen-free flame resistant polyethylene extrusion coated paper facings. Prog. Org. Coat. 2015, 78, 67-72.

(36) Jing, X. K.; Wang, X. S.; Guo, D. M.; Zhang, Y.; Zhai, F. Y.; Wang, X. L.; Chen, L.; Wang, Y. Z. The high-temperature self-crosslinking contribution of azobenzene groups 
to the flame retardance and anti-dripping of copolyesters. J. Mater. Chem. A. 2013, 1, 9264-9272.

(37) Wang, Q.; Wu, J. W.; Gao, Y. S.; Zhang, Z.; Wang, J. Y.; Zhang, X.; Yan, X. R.; Umar, A.; Guo, Z. H.; O'Hare, D. Polypropylene/Mg3Al-tartrazine LDH nanocomposites with enhanced thermal stability, UV absorption, and rheological properties. RSC Adv. 2013, 3, 26017-26024.

(38) Adachipagano, M.; Forano, C.; Besse, J. P. Synthesis of Al-rich hydrotalcite-like compounds by using the urea hydrolysis reaction-control of size and morphology. J. Mater. Chem. 2003, 13, 1988-1993.

(39) Huang, S.; Cen, X.; Peng, H. D.; Guo, S. Z.; Wang, W. Z.; Liu, T. X. Heterogeneous Ultrathin Films of Poly(vinyl alcohol)/Layered Double Hydroxide and Montmorillonite Nanosheets via Layer-by-Layer Assembly. J. Phys. Chem. B. 2009, 113, 15225-15230.

(40) He, L. H.; Wang, G. M.; Tang, Q.; Fu, X. K.; Gong, C. B. Synthesis and characterization of novel electrochromic and photoresponsive materials based on azobenzene-4,4'dicarboxylic acid dialkyl ester. J. Mater. Chem. C. 2014, 2, 8162-8169.

(41) Kang, N. J.; Wang, D. Y. A green functional nanohybrid: preparation, characterization and properties of a b-cyclodextrin based functional layered double hydroxide. J. Mater. Chem. A. 2013, 1, 11376-11383.

(42) Manzi-Nshuti, C.; Songtipya, P.; Manias, E.; Jimenez-Gasco, M. d. M.; Hossenlopp, J. M.; Wilkie, C. A. Polymer nanocomposites using zinc aluminum and magnesium aluminum oleate layered double hydroxides: Effects of the polymeric compatibilizer and of composition on the thermal and fire properties of PP/LDH nanocomposites. Polym. Degrad. Stab. 2009, 94, 2042-2054.

(43) Nagendra, B.; Mohan, K.; Gowd, E. B. Polypropylene/Layered Double Hydroxide (LDH) Nanocomposites: Influence of LDH Particle Size on the Crystallization Behavior of Polypropylene. ACS Appl. Mat. Interfaces. 2015, 7, 12399-12410.

(44) Mallakpour, S.; Dinari, M.; Hatami, M. Modification of Mg/Al-layered double hydroxide with l-aspartic acid containing dicarboxylic acid and its application in the enhancement of the thermal stability of chiral poly(amide-imide). RSC Adv. 2014, 4, 42114-42121.

(45) Ramesh Babu, R.; Kumaresan, S.; Vijayan, N.; Gunasekaran, M.; Gopalakrishnan, R.; Kannan, P.; Ramasamy, P. Growth of 4,4'-dihydroxyazobenzene (DHAB) and its characterization. J. Cryst. Growth. 2003, 256, 387-392.

(46) Langford, S. A.; Sugden, J. K.; Fitzpatrick, R. W. Detection and determination of the hydrazo and azo photoproducts of 4-aminobenzoic acid by high-performance liquid chromatography. J. Pharm. Biomed. Anal. 1996, 14, 1615-1623.

(47) Huang, G. B.; Chen, S. Q.; Song, P. A.; Lu, P. P.; Wu, C. L.; Liang, H. D. Combination effects of graphene and layered double hydroxides on intumescent flame-retardant poly(methyl methacrylate) nanocomposites. Appl. Clay Sci. 2013, 88, 78-85. 
(48) Zeng, H. Y.; Du, J. Z.; Xu, S.; Liao, M. C.; Liu, X. J.; Duan, H. Z.; Chen, C. R. Influences of a glycerin co-solvent on the compatibility of MgAl hydrotalcites with a polypropylene matrix. RSC Adv. 2015, 5, 64814-64820.

(49) Alansi, A. M.; Alkayali, W. Z.; Al-qunaibit, M. H.; Qahtan, T. F.; Saleh, T. A. Synthesis of exfoliated polystyrene/anionic clay MgAl-layered double hydroxide: structural and thermal properties. RSC Adv. 2015, 5, 71441-71448.

(50) Du, B. X.; Guo, Z. H.; Fang, Z. P. Effects of organo-clay and sodium dodecyl sulfonate intercalated layered double hydroxide on thermal and flame behaviour of intumescent flame retarded polypropylene. Polym. Degrad. Stab. 2009, 94, 1979-1985.

(51) Kalali, E. N.; Wang, X.; Wang, D. Y. Functionalized layered double hydroxide-based epoxy nanocomposites with improved flame retardancy and mechanical properties. $J$. Mater. Chem. A. 2015, 3, 6819-6826.

(52) Wang, X.; Kalali, E. N.; Wang, D. Y. Renewable cardanol-based surfactant modified layered double hydroxide as a flame retardant for epoxy resin. ACS Sustain. Chem. Eng. 2015, 3, 3281-3290.

(53) Schartel, B.; Hull, T. R. Development of fire-retarded materials-Interpretation of cone calorimeter data. Fire. Mater. 2007, 31, 327-354.

(54) Dong, Y. Y.; Gui, Z.; Hu, Y.; Wu, Y.; Jiang, S. H. The influence of titanate nanotube on the improved thermal properties and the smoke suppression in poly(methyl methacrylate). J. Hazard. Mater. 2012, 209, 34-39.

(55) Chow, W. S.; Teoh, E. L. Flexible and flame resistant poly(lactic acid)/ organomontmorillonite nanocomposites. J. Appl. Polym. Sci. 2015, 132, 41253, doi: 10.1002/app.41253.

(56) Nicolas, R. C.; Wilén, C. E.; Roth, M.; Pfaendner, R.; King, R. E. Azoalkanes: A Novel Class of Flame Retardants. Macromol. Rapid. Comm. 2006, 27, 976-981.

(57) Isitman, N. A.; Kaynak, C. Nanoclay and carbon nanotubes as potential synergists of an organophosphorus flame-retardant in poly(methyl methacrylate). Polym. Degrad. Stab. 2010, 95, 1523-1532. 


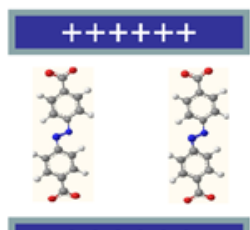

$+++++$

NiZnAl-LDH

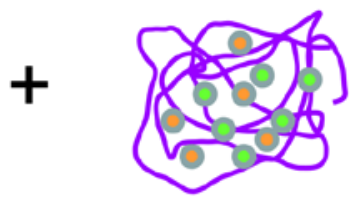

PP/IFR System
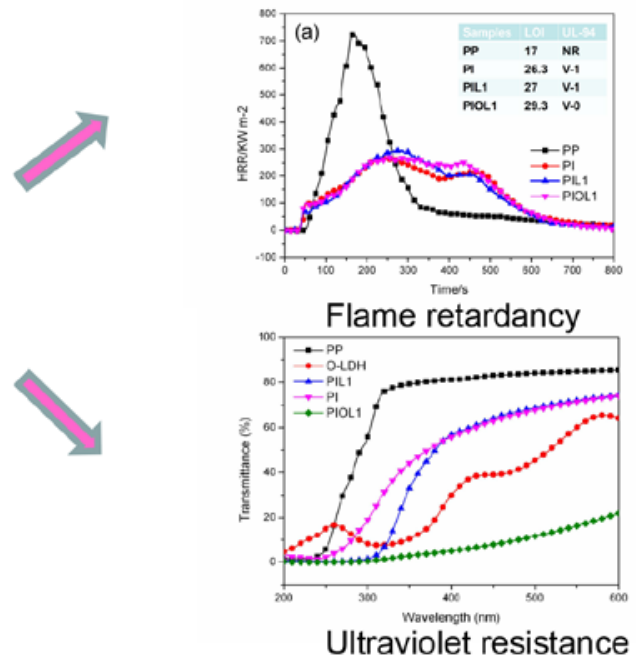

Azobenzene-4,4'-dicarboxylic acid (Azo-DBA) modified LDH (O-LDH) has been successfully prepared by ion exchange method. After introduction of $1 \mathrm{wt} \% \mathrm{O}-\mathrm{LDH}$, it can be found that the PP/IFR/O-LDH (PIOL1) shows a $78.0 \%$ and $72.8 \%$ reduction in the fire growth rate index (FIGRA) and ultraviolet transmission intensity, respectively compared with neat PP.

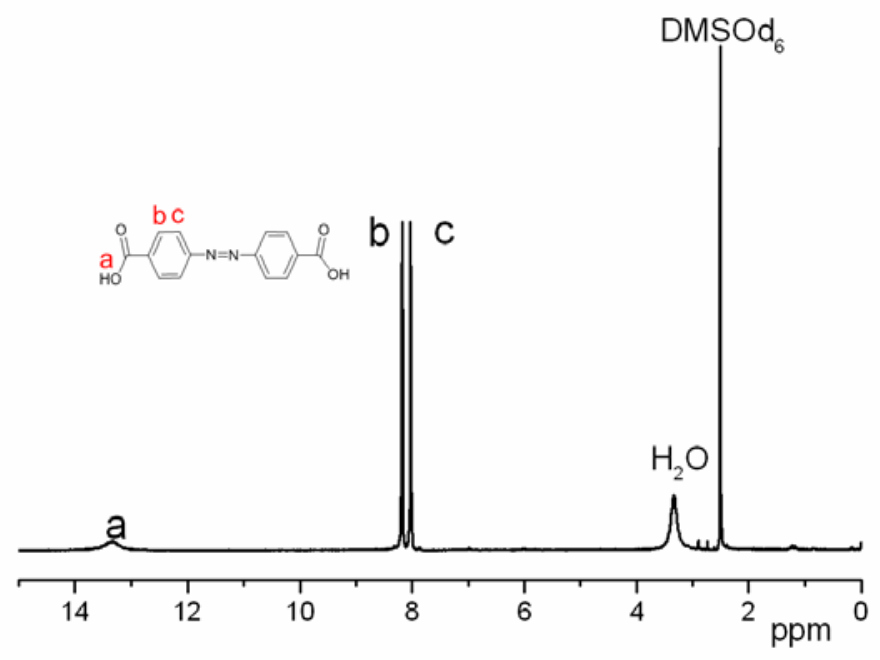

Figure S1 the ${ }^{1} \mathrm{H}-\mathrm{NMR}$ of Azo-DBA 